\title{
Unexpected Photoisomerization of a Pincer-type Amido Ligand Leads to Facial Coordination at Pt(IV).
}

\author{
Seth B. Harkins and Jonas C. Peters \\ Division of Chemistry and Chemical Engineering, Arnold and Mabel Beckman Laboratories of \\ Chemical Synthesis, California Institute of Technology, Pasadena, California 91125.
}

\section{Supporting Information}

S-2 Experimental Section

Figure 1: Fully Labeled Drawing of (mer-BQA)PtMe 2 I, 2

S-5 Figure 2: Fully Labeled Drawing of (fac-BQA)PtMe ${ }_{2} \mathrm{I}, 3$

S-6 Figure 3: Fully Labeled Drawing of [H(fac-BQA)PtMe $\left.\mathrm{I}_{2}\right]\left[\mathrm{BF}_{4}\right], 4$

S-7 Table 1. Crystal Data and Structure Refinement for 2

S-8 Table 2. Atomic Coordinates and Equivalent Isotropic Displacement Parameters for 2

S-9 Table 3. Bond Lengths and Angles for 2

S-11 Table 4. Anisotropic Parameters for 2

S-12 Table 5. Hydrogen Coordinates and Isotropic Displacement Parameters for 2

S-13 Table 6. Crystal Data and Structure Refinement for 3

S-14 Table 7. Atomic Coordinates and Equivalent Isotropic Displacement Parameters for 3

S-15 Table 8. Bond Lengths and Angles for 3

S-17 Table 9. Anisotropic Parameters for 3

S-18 Table 10. Hydrogen Coordinates and Isotropic Displacement Parameters for 3

S-19 Table 11. Crystal Data and Structure Refinement for 4

S-20 Table 12. Atomic Coordinates and Equivalent Isotropic Displacement Parameters for 4

S-21 Table 13. Bond Lengths and Angles for 4

S-22 Table 14. Anisotropic Parameters for 4

S-22 Table 15. Hydrogen Coordinates and Isotropic Displacement Parameters for 4

S-22 Table 16. Hydrogen bonds for 4 
General: All manipulations were carried out using standard Schlenk or glove box techniques under a dinitrogen atmosphere. Unless otherwise noted, solvents were deoxygenated and dried thoroughly by sparging with $\mathrm{N}_{2}$ gas followed by passage through an activated alumina column. Non-halogenated solvents were typically tested with a standard purple solution of sodium benzophenone ketyl in tetrahydrofuran to confirm effective oxygen and moisture removal. The preparation of (BQA)PtMe was previously reported. ${ }^{1}$ Other reagents were purchased from commercial vendors and used without further purification. Elemental analyses were performed by Desert Analytics, Tucson, Az. A Varian Mercury-300 NMR spectrometer or a Varian Inova-500 NMR spectrometer was used to record ${ }^{1} \mathrm{H},{ }^{2} \mathrm{H},{ }^{13} \mathrm{C}$, and ${ }^{19} \mathrm{~F}$ NMR spectra. 1D-NOE experiments were conducted using the Varian GLIDE software package. ${ }^{1} \mathrm{H}$ and ${ }^{13} \mathrm{C}$ NMR chemical shifts were referenced to residual solvent. ${ }^{19} \mathrm{~F}$ NMR chemical shifts were referenced to an external $\mathrm{C}_{6} \mathrm{~F}_{6}$ sample with a chemical shift of $-165 \mathrm{ppm}$. Deuterated solvents were purchased from Cambridge Isotope Labs and were degassed and dried over activated $3 \AA$ molecular sieves prior to use. Uv-vis spectra were collected on a Cary 50 spectrophotometer in $\mathrm{CH}_{2} \mathrm{Cl}_{2}$ with $1 \mathrm{~cm}$ quartz cuvettes. IR measurements were obtained with a $\mathrm{KBr}$ solution cell using a Bio-Rad Excalibur FTS 3000 spectrometer controlled by Bio-Rad Merlin Software (v. 2.97) set at $4 \mathrm{~cm}^{-1}$ resolution. X-ray diffraction studies were carried out at the Beckman Institute Crystallographic Facility on a Brüker Smart 1000 CCD diffractometer and solved using SHELX v. 6.14.

Synthesis of (mer-BQA)Pt(CH $\mathbf{C H}_{2} \mathbf{I}$ / (mer-BQA)Pt $\left(\mathbf{C H}_{3}\right)\left(\mathbf{C D}_{3}\right) \mathbf{I}$, 2: A solution of (BQA)PtMe (400 mg, 0.833 $\mathrm{mmol}), \mathrm{CH}_{3} \mathrm{I}(103 \mu \mathrm{L}, 1.67 \mathrm{mmol})$, and acetone $(50 \mathrm{~mL})$ were combined in a glass reaction bomb, sealed with its Teflon stopcock, and heated to $70{ }^{\circ} \mathrm{C}$ for $18 \mathrm{~h}$ in the absence of light. The purple solution was evaporated to dryness under reduced pressure, and washed with petroleum ether $(2 \times 40 \mathrm{~mL})$. Drying in vacuo of the powdery-purple solid (470 mg, 92\%) yield a spectroscopically pure product. X-ray quality crystals were obtained by vapor diffusion of petroleum ether into acetone. Reactions with $\mathrm{CD}_{3} \mathrm{I}$ proceeded under identical conditions. Characterization data for the all protio form: ${ }^{1} \mathrm{H}$ NMR $\left(\mathrm{CD}_{2} \mathrm{Cl}_{2}, 300 \mathrm{MHz}, 25^{\circ} \mathrm{C}\right): \delta 8.71\left(\mathrm{~m},{ }^{2} J_{\mathrm{PtH}}=41 \mathrm{~Hz}, 2 \mathrm{H}\right), 8.30(\mathrm{~d}, 2 \mathrm{H}), 7.77(\mathrm{~d}, 2 \mathrm{H})$, $7.66(\mathrm{t}, 2 \mathrm{H}), 7.48(\mathrm{~m}, 2 \mathrm{H}), 7.16(\mathrm{~d}, 2 \mathrm{H}), 1.67\left(\mathrm{~s},{ }^{2} \mathrm{~J}_{\mathrm{PtH}}=60 \mathrm{~Hz}, 3 \mathrm{H}\right), 1.43\left(\mathrm{~s},{ }^{2} \mathrm{~J}_{\mathrm{PtH}}=70 \mathrm{~Hz}, 3 \mathrm{H}\right) .{ }^{13} \mathrm{C}$ NMR $\left(\mathrm{CD}_{2} \mathrm{Cl}_{2}\right.$, $\left.126 \mathrm{MHz}, 25{ }^{\circ} \mathrm{C}\right): \delta 155.1,146.7,140.3,139.4,133.4,130.9,122.3,115.7,115.6,15.2\left({ }^{1} J_{\mathrm{PtC}}=550 \mathrm{~Hz}\right), 1.89\left({ }^{1} J_{\mathrm{PtC}}=\right.$ $582 \mathrm{~Hz})$. UV-vis (nm $\left.\left(\varepsilon \mathrm{M}^{-1} \mathrm{~cm}^{-1}\right), \mathrm{CH}_{2} \mathrm{Cl}_{2}\right): 292$ (30000), 300 (27100), sh 396 (2900), 388 (3400), 534 (15500). IR $\left(\mathrm{cm}^{-1}, \mathrm{KBr}\right): 3053(\mathrm{vw}), 2902(\mathrm{w}), 1582(\mathrm{~m}), 1564$ (s), 1496 (s), 1463 (s), $1400(\mathrm{~s}), 1225(\mathrm{w}), 1180(\mathrm{~m}), 1134(\mathrm{~m})$, $813(\mathrm{~m}), 770(\mathrm{~m}), 736(\mathrm{~m})$. Anal. Calcd. for $\mathrm{C}_{20} \mathrm{H}_{18} \mathrm{IN}{ }_{3} \mathrm{Pt}$ : C, 38.60; H, 2.92; N, 6.75. Found: C, 38.76; H, 3.15, N, 6.51 .

Synthesis of (fac-BQA)Pt( $\left(\mathbf{C H}_{3}\right)_{2} \mathbf{I} /\left(\right.$ fac-BQA)Pt $\left(\mathbf{C H}_{3}\right)\left(\mathbf{C D}_{3}\right) \mathbf{I}$, 3: A solution of 2 (196 $\left.\mathrm{mg}, 0.315 \mathrm{mmol}\right)$ in acetone $(20 \mathrm{~mL})$ was added to a $200 \mathrm{~mL}$ reaction bomb, sealed with its Teflon stopcock, and placed 2" underneath a 100 watt incandescent light bulb for $48 \mathrm{~h}$, with periodic agitation. During this period, the solution went from purple to red in color. The solvent was removed under reduced pressure affording a red solid which was washed with petroleum ether and dried in vacuo (196 mg, >99\%). Quantitative conversion was observed by NMR spectroscopy. Reactions with (mer-BQA)Pt $\left(\mathrm{CH}_{3}\right)\left(\mathrm{CD}_{3}\right)$ I proceeded under identical conditions. Characterization data for the all protio form: ${ }^{1} \mathrm{H}$ NMR $\left(\mathrm{CD}_{2} \mathrm{Cl}_{2}, 300 \mathrm{MHz}, 25{ }^{\circ} \mathrm{C}\right): \delta 9.80\left(\mathrm{~m},{ }^{3} J_{\mathrm{PtH}}=17 \mathrm{~Hz}, 2 \mathrm{H}\right), 8.25(\mathrm{~m}, 2 \mathrm{H}), 7.96(\mathrm{~d}, 2 \mathrm{H}), 7.58-$ $7.47(\mathrm{~m}, 4 \mathrm{H}), 7.40(\mathrm{~d}, 2 \mathrm{H}), 1.25\left(\mathrm{~s}\left\{\mathrm{Pt}_{-} \mathrm{CH}_{3}\right\},{ }^{2} \mathrm{~J}_{\mathrm{PtH}}=71 \mathrm{~Hz}, 6 \mathrm{H}\right) .{ }^{13} \mathrm{C} \mathrm{NMR}\left(\mathrm{CD}_{2} \mathrm{Cl}_{2}, 126 \mathrm{MHz}, 25{ }^{\circ} \mathrm{C}\right): \delta 159.7$, 150.2, 147.1, 138.3, 131.1, 129.1, 123.4, 122.7, 121.2, $-8.0\left({ }^{1} J_{\mathrm{PtC}}=602 \mathrm{~Hz}\right)$. UV-vis $\left(\mathrm{nm}\left(\varepsilon^{-1} \mathrm{~cm}^{-1}\right), \mathrm{CH}_{2} \mathrm{Cl}_{2}\right): 284$ (15900), sh 298 (10600), sh 325 (4000), 422 (4700), sh 502 (1300). IR ( $\left.\mathrm{cm}^{-1}, \mathrm{KBr}\right): 3051(\mathrm{w}), 2962$ (w), 2897 (m), 1575 (m), 1565 (m), 1497 (s), 1458 (s), 1374 (s), 1308 (m), 1256 (m), 1224 (m), 1113 (m), 1056 (w), 1031 (w), 841 (w), $810(\mathrm{~m}), 772(\mathrm{~m})$. Anal. Calcd. for $\mathrm{C}_{20} \mathrm{H}_{18} \mathrm{IN}_{3} \mathrm{Pt}$ : C, 38.60; H, 2.92; N, 6.75. Found: C, 38.81; H, 3.18, N, 6.50.

Synthesis of $\left[\mathbf{H}(\mathbf{f a c}-\mathbf{B Q A}) \mathbf{P t}\left(\mathbf{C H}_{3}\right)_{2} \mathbf{I}\right]\left[\mathbf{B F}_{4}\right]$, 4. A $54 \mathrm{wt} \%$ solution of $\mathrm{HBF}_{4}$ in ether $(19 \mu \mathrm{L}, 0.137 \mathrm{mmol})$ was added to a solution of $4(81 \mathrm{mg}, 0.130 \mathrm{mmol})$ in $\mathrm{CH}_{2} \mathrm{Cl}_{2}(7 \mathrm{~mL})$ in a glass scintillation vial. The reaction was stirred for $2 \mathrm{~h}$ at $25^{\circ} \mathrm{C}$ and the product was precipitated by addition of petroleum ether $(13 \mathrm{~mL})$. The rose colored solid $(90$ $\mathrm{mg}, 97 \%$ ) was collected on a fritted glass funnel and dried in vacuo. The product was found to be pure by ${ }^{1} \mathrm{H}$ NMR spectroscopy and micro analysis. Crystals suitable for X-ray diffraction were obtained by diffusion of petroleum ether in to a concentrated solution of 4 in THF. ${ }^{1} \mathrm{H}$ NMR $\left(\mathrm{CD}_{2} \mathrm{Cl}_{2}, 300 \mathrm{MHz}, 25{ }^{\circ} \mathrm{C}\right): \delta 10.33\left(\mathrm{~s}\{\mathrm{~N}-\mathrm{H}\},{ }^{2} J_{\mathrm{PtH}}=21\right.$ $\mathrm{Hz}, 1 \mathrm{H}), 9.90\left(\mathrm{~m},{ }^{3} J_{\mathrm{PtH}}=17 \mathrm{~Hz}, 2 \mathrm{H}\right), 8.78(\mathrm{~d}, 2 \mathrm{H}), 8.50(\mathrm{~m}, 2 \mathrm{H}), 7.96(\mathrm{~m}, 2 \mathrm{H}), 7.83(\mathrm{t}, 2 \mathrm{H}), 7.74(\mathrm{~m}, 2 \mathrm{H}) 1.54(\mathrm{~s}$ $\left.\left\{\mathrm{Pt}_{-\mathrm{CH}_{3}}\right\},{ }^{2} J_{\mathrm{PtH}}=70 \mathrm{~Hz}, 6 \mathrm{H}\right) .{ }^{13} \mathrm{C}$ NMR $\left(\mathrm{CD}_{2} \mathrm{Cl}_{2}, 126 \mathrm{MHz}, 25{ }^{\circ} \mathrm{C}\right): \delta 152.0,144.8,143.7,139.9,131.5,130.5$, 130.1, 129.9, 125.0, -12.41 ( $\left.{ }^{1} J_{\mathrm{PtC}}=514 \mathrm{~Hz}\right) .{ }^{19} \mathrm{~F} \mathrm{NMR}\left(\mathrm{CD}_{2} \mathrm{Cl}_{2}, 282 \mathrm{MHz}, 25^{\circ} \mathrm{C}\right): \delta-147.4\left(\mathrm{~d},{ }^{1} J_{\mathrm{BF}}=15 \mathrm{~Hz}\right) . \mathrm{UV}$-vis (nm $\left.\left(\varepsilon \mathrm{M}^{-1} \mathrm{~cm}^{-1}\right), \mathrm{CH}_{2} \mathrm{Cl}_{2}\right): 302$ (12900), 317 (10000), sh 240 (1700), 510 (630). IR (cm $\left.{ }^{-1}, \mathrm{KBr}\right): 3077$ (br, N-H),

\footnotetext{
${ }^{1}$ Harkins, S. B.; Peters, J. C. Organometallics 2002, 21, 1753.
} 
2978 (w), 2903 (w), 1510 (s), 1467 (m), 1398 (m), 1358 (m), 1221 (w), 1081 (s br, B-F), 835 (m), 798 (m), 769 (m) 734 (w). IR ( $\mathrm{cm}^{-1}, \mathrm{CH}_{2} \mathrm{Cl}_{2}$ sol. in KBr): 3127 (br, N-H), 2910 (w), 1591 (w), 1567 (w), 15131467 (w), 1359 (m), 1080 (s br), 1000 (m), 835 (m). Anal. Calcd. for $\mathrm{C}_{20} \mathrm{H}_{19} \mathrm{BF}_{4} \mathrm{IN}_{3} \mathrm{Pt}$ : C, 33.82; H, 2.70; N, 5.92. Found: C, 33.84; H, $2.46, \mathrm{~N}, 5.73$.

Conversion of (BQA)Pt-Me to (BQA)Pt-Ph at rt: Under inert atmosphere an NMR tube was charged with 1 (20.0 $\mathrm{mg}, 0.042 \mathrm{mmol}), \mathrm{B}\left(\mathrm{C}_{6} \mathrm{~F}_{5}\right)_{3}(21.3 \mathrm{mg}, \quad 0.042 \mathrm{mmol})$, and $\mathrm{C}_{6} \mathrm{D}_{6}(\sim 0.6 \mathrm{~mL}) .{ }^{1} \mathrm{H} \mathrm{NMR}$ spectral analysis of the solution showed complete loss of the starting material methyl complex and quantitative production of previously characterized (BQA)Pt-Ph. ${ }^{1}$ 
Figure 1: Fully Labeled Drawing of (mer-BQA)PtMe 2 I, 2 (hydrogen atoms omitted for clarity).

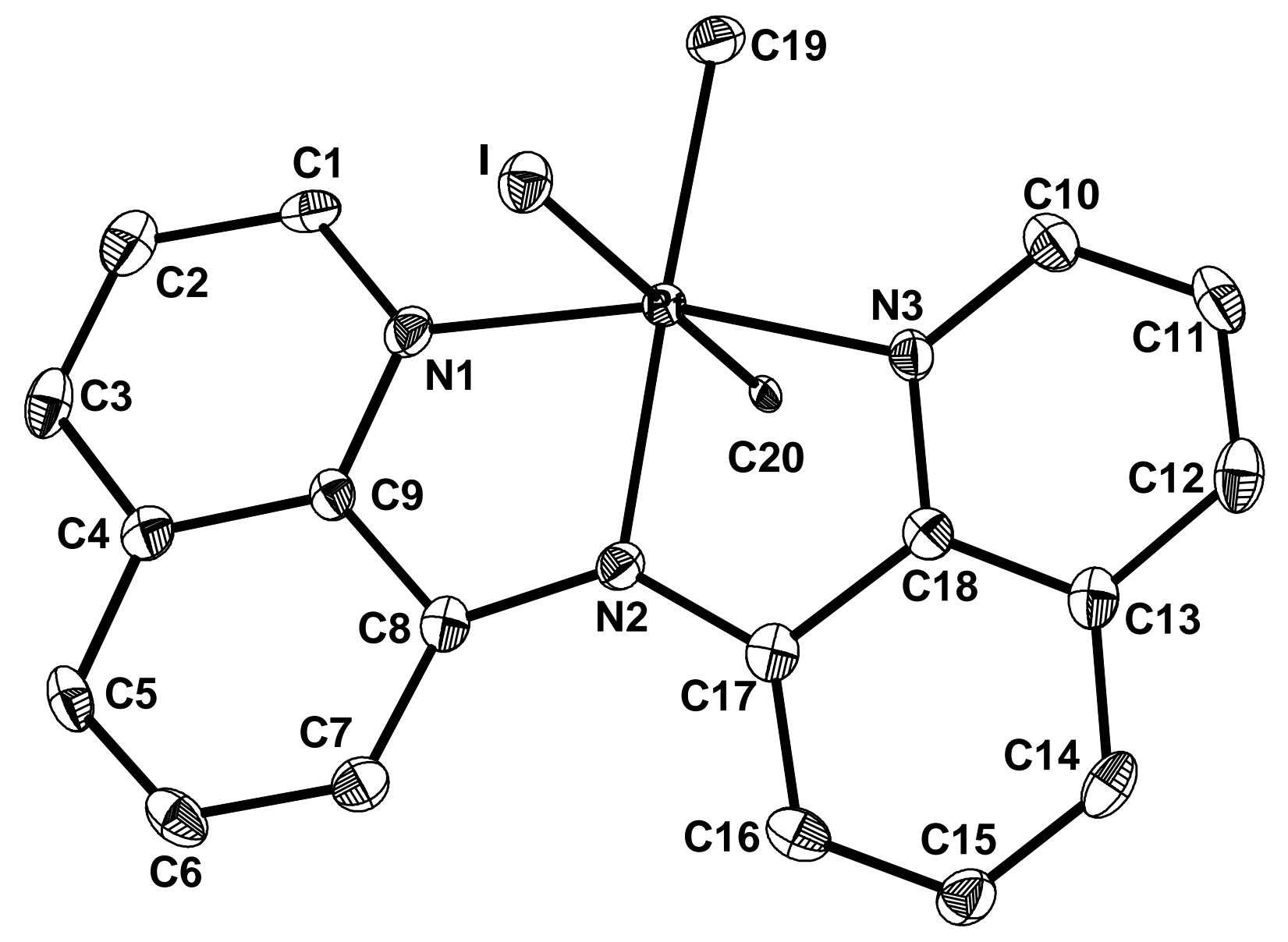


Figure 2: Fully Labeled Drawing of (fac-BQA)PtMe $\mathrm{I}_{2}, 3$ (hydrogen atoms omitted for clarity).

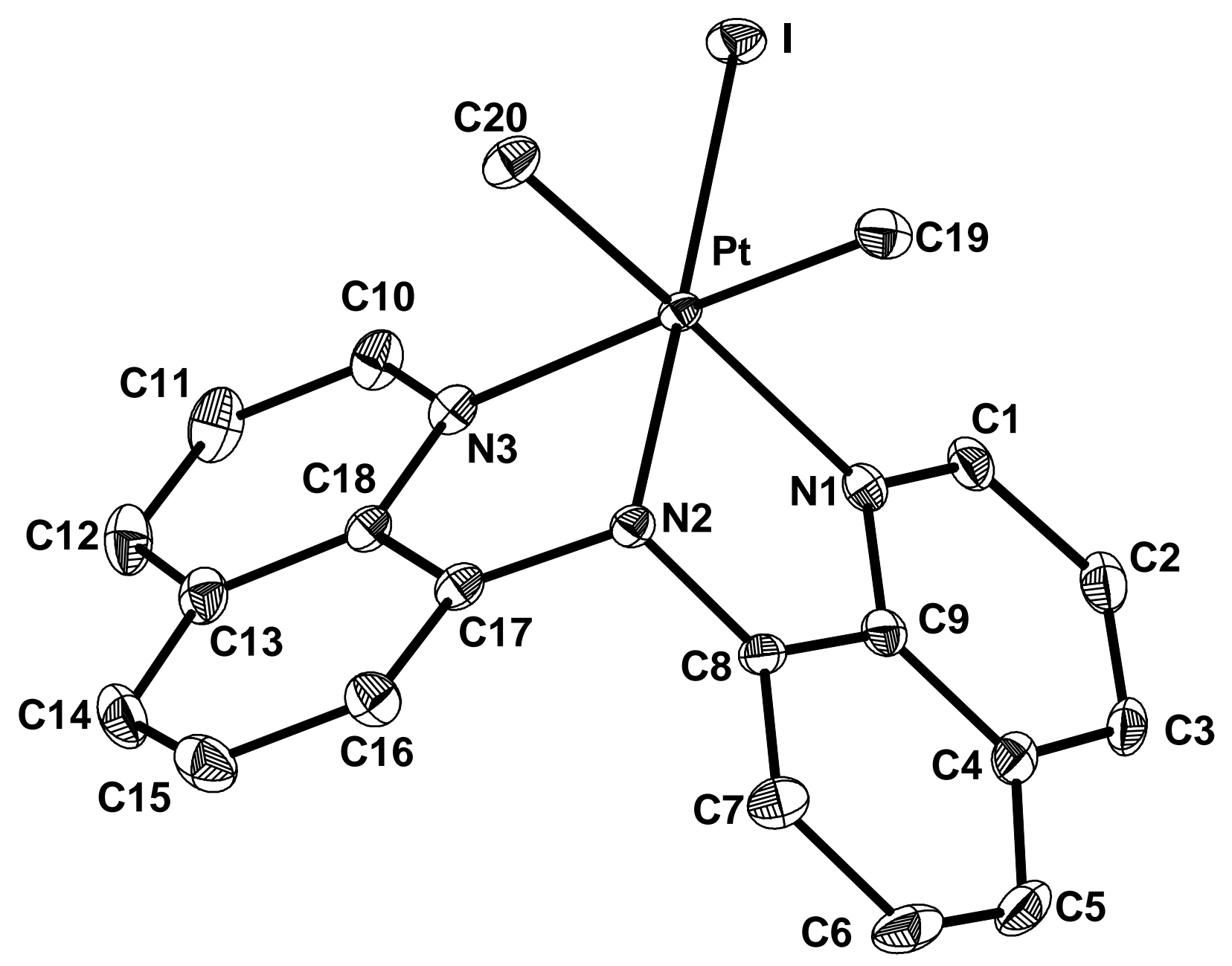


Figure 3: Fully Labeled Drawing of [H(fac-BQA)PtMe $\left.\mathrm{P}_{2}\right]\left[\mathrm{BF}_{4}\right], 4$ (hydrogen atoms attached to $\mathrm{C}$ omitted for clarity). Italicized atom labels correspond to atoms generated by symmetry transformation $\{\mathrm{x},-\mathrm{y}+1 / 2, \mathrm{z}\}$.

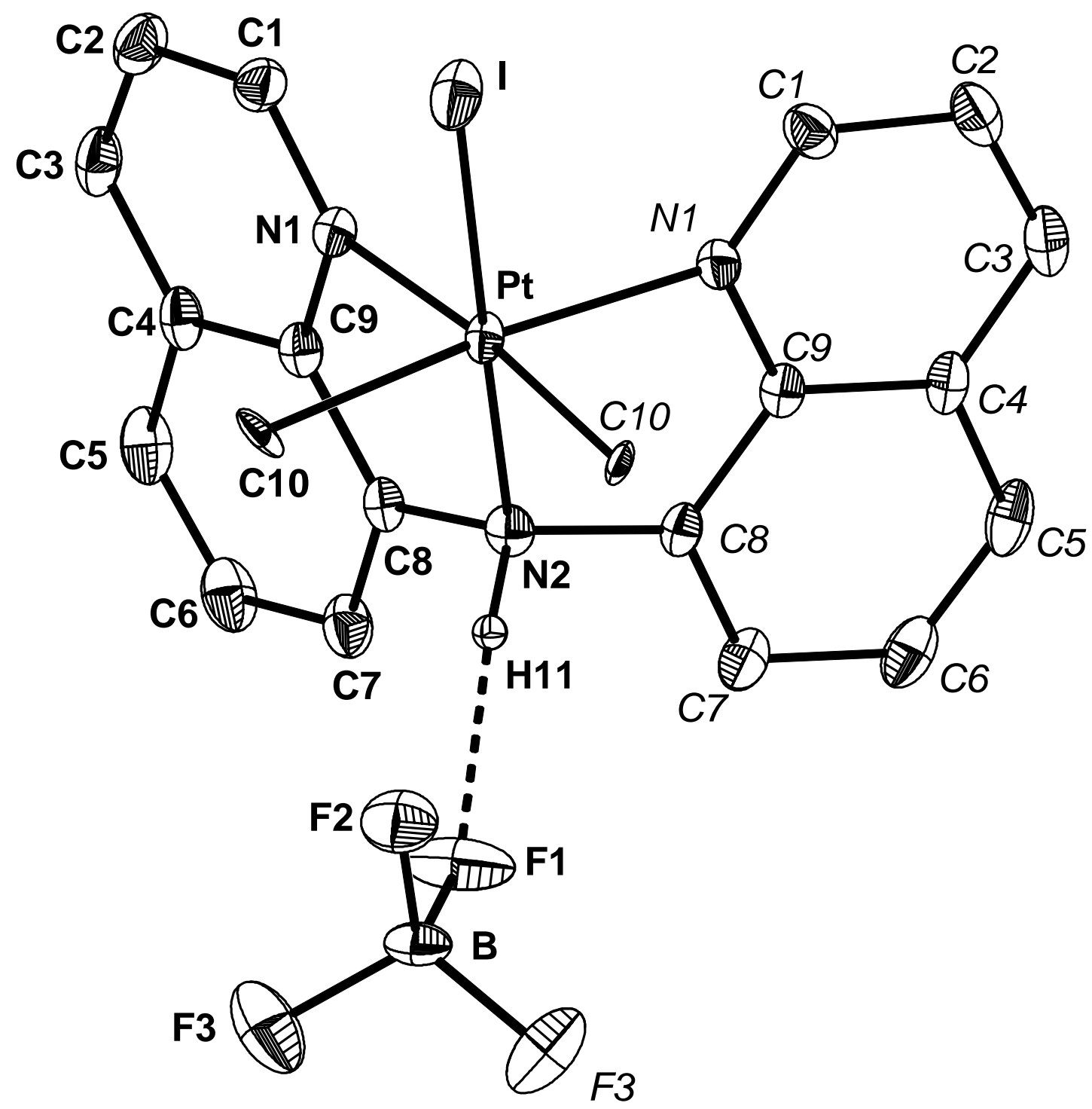


Table 1. Crystal data and structure refinement for $\mathbf{2}$.

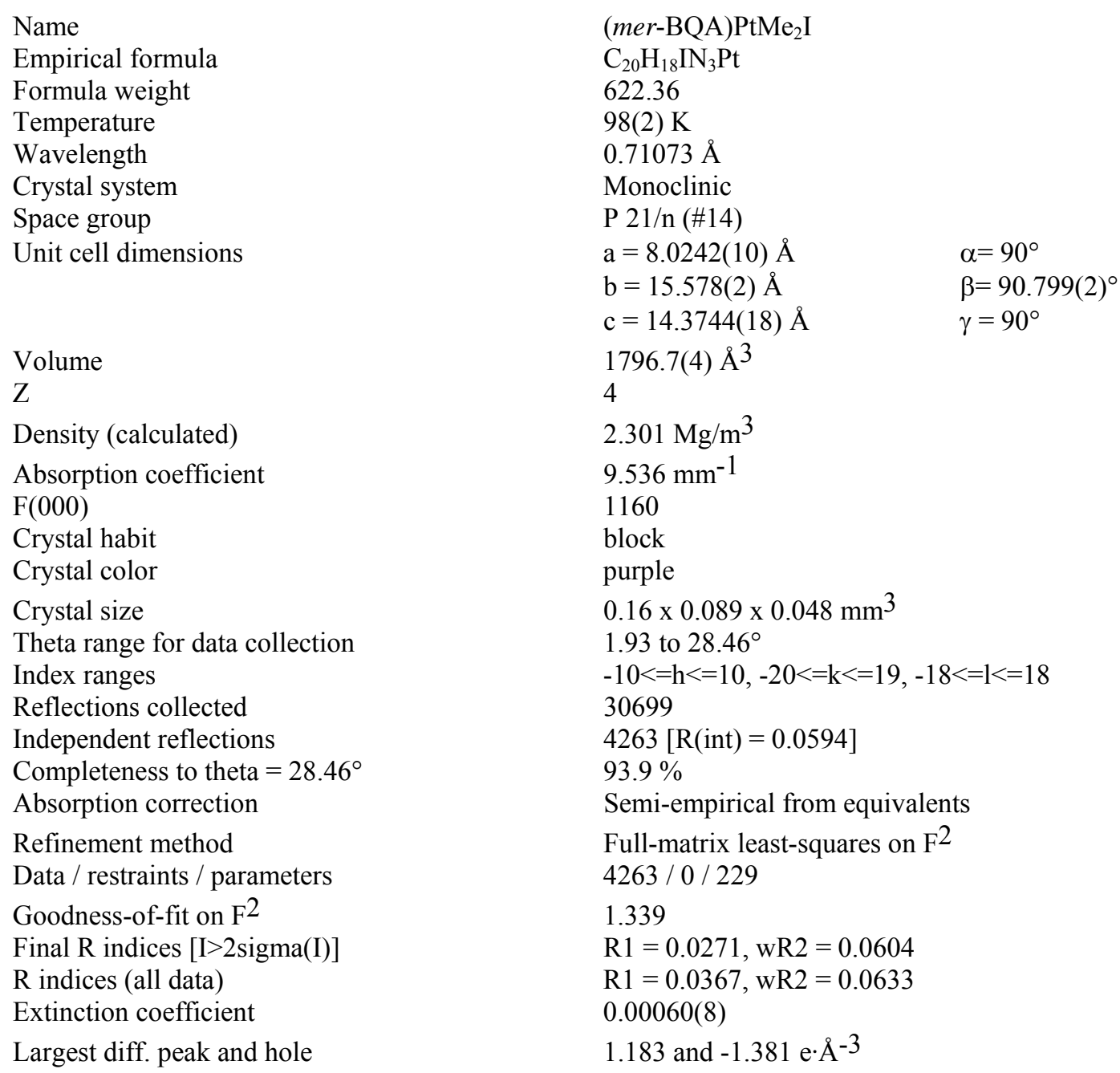

\section{Refinement Details}

Refinement of $\mathrm{F}^{2}$ against all reflections. The weighted $\mathrm{R}$-factor $\mathrm{wR}$ and goodness of fit $\mathrm{S}$ are based on $\mathrm{F}^{2}$, conventional $R$-factors $R$ are based on $F$, with $F$ set to zero for negative $F^{2}$. The threshold expression of $F^{2}>$ $2 \operatorname{sigma}\left(\mathrm{F}^{2}\right)$ is used only for calculating $\mathrm{R}$-factors $(\mathrm{gt})$ etc. and is not relevant to the choice of reflections for refinement. R-factors based on $\mathrm{F}^{2}$ are statistically about twice as large as those based on $\mathrm{F}$, and $\mathrm{R}$ - factors based on all data will be even larger.

All esds (except the esd in the dihedral angle between two 1.s. planes) are estimated using the full covariance matrix. The cell esds are taken into account individually in the estimation of esds in distances, angles and torsion angles; correlations between esds in cell parameters are only used when they are defined by crystal symmetry. An approximate (isotropic) treatment of cell esds is used for estimating esds involving l.s. planes.

A slightly large prolate is evident in thermal ellipsoid of $\mathrm{C} 20$ which results from a partial population of I in this position arising from a trace quantity of the mirror image of $\mathbf{2}$ which has crystallized in an errant conformation. Refinement with a partial population of I in this position $(<3 \%)$ did not significantly improve the overall refinement and was not included as a part of the final refinement. 
Table 2. Atomic coordinates ( $\left.\mathrm{x} 10^{4}\right)$ and equivalent isotropic displacement parameters $\left(\AA^{2} \times 10^{3}\right)$ for 2 . $U(e q)$ is defined as one third of the trace of the orthogonalized $U^{i j}$ tensor.

\begin{tabular}{lrrrr}
\hline & $\mathrm{x}$ & $\mathrm{y}$ & $\mathrm{z}$ & $\mathrm{U}(\mathrm{eq})$ \\
\hline $\mathrm{Pt}$ & $1823(1)$ & $1654(1)$ & $2458(1)$ & $11(1)$ \\
$\mathrm{I}$ & $404(1)$ & $3272(1)$ & $2233(1)$ & $18(1)$ \\
$\mathrm{N}(1)$ & $1820(5)$ & $1523(2)$ & $1068(3)$ & $15(1)$ \\
$\mathrm{N}(2)$ & $-458(4)$ & $1103(2)$ & $2292(3)$ & $13(1)$ \\
$\mathrm{N}(3)$ & $1195(5)$ & $1597(2)$ & $3810(3)$ & $14(1)$ \\
$\mathrm{C}(1)$ & $3049(6)$ & $1705(3)$ & $483(3)$ & $17(1)$ \\
$\mathrm{C}(2)$ & $2858(6)$ & $1627(3)$ & $-482(3)$ & $21(1)$ \\
$\mathrm{C}(3)$ & $1358(6)$ & $1357(3)$ & $-839(3)$ & $18(1)$ \\
$\mathrm{C}(4)$ & $27(6)$ & $1161(3)$ & $-246(3)$ & $15(1)$ \\
$\mathrm{C}(5)$ & $-1560(6)$ & $898(3)$ & $-579(3)$ & $17(1)$ \\
$\mathrm{C}(6)$ & $-2768(6)$ & $715(3)$ & $47(3)$ & $19(1)$ \\
$\mathrm{C}(7)$ & $-2500(6)$ & $762(3)$ & $1008(3)$ & $17(1)$ \\
$\mathrm{C}(8)$ & $-960(5)$ & $1011(3)$ & $1383(3)$ & $14(1)$ \\
$\mathrm{C}(9)$ & $311(5)$ & $1235(3)$ & $727(3)$ & $13(1)$ \\
$\mathrm{C}(10)$ & $2047(6)$ & $1881(3)$ & $4547(3)$ & $18(1)$ \\
$\mathrm{C}(11)$ & $1466(6)$ & $1769(3)$ & $5458(3)$ & $21(1)$ \\
$\mathrm{C}(12)$ & $5(6)$ & $1348(3)$ & $5594(3)$ & $20(1)$ \\
$\mathrm{C}(13)$ & $-910(6)$ & $1030(3)$ & $4825(3)$ & $16(1)$ \\
$\mathrm{C}(14)$ & $-2413(6)$ & $563(3)$ & $4928(3)$ & $18(1)$ \\
$\mathrm{C}(15)$ & $-3193(6)$ & $244(3)$ & $4162(3)$ & $20(1)$ \\
$\mathrm{C}(16)$ & $-2601(5)$ & $389(3)$ & $3255(3)$ & $17(1)$ \\
$\mathrm{C}(17)$ & $-1192(5)$ & $876(3)$ & $3110(3)$ & $14(1)$ \\
$\mathrm{C}(18)$ & $-300(5)$ & $1176(3)$ & $3930(3)$ & $13(1)$ \\
$\mathrm{C}(19)$ & $4204(6)$ & $2171(3)$ & $2647(3)$ & $17(1)$ \\
$\mathrm{C}(20)$ & $2928(5)$ & $438(3)$ & $2607(3)$ & $18(1)$ \\
& & & & \\
\hline & & & & \\
\hline
\end{tabular}


Table 3. Bond lengths $[\AA]$ and angles $\left[{ }^{\circ}\right]$ for 2 .

\begin{tabular}{|c|c|c|c|}
\hline Pt-N(1) & $2.008(4)$ & $\mathrm{N}(2)-\mathrm{Pt}-\mathrm{C}(19)$ & $177.62(16)$ \\
\hline Pt-N(3) & $2.016(4)$ & $\mathrm{N}(1)-\mathrm{Pt}-\mathrm{C}(20)$ & $90.22(16)$ \\
\hline $\mathrm{Pt}-\mathrm{N}(2)$ & $2.033(4)$ & $\mathrm{N}(3)-\mathrm{Pt}-\mathrm{C}(20)$ & $88.41(16)$ \\
\hline Pt-C(19) & $2.087(4)$ & N(2)-Pt-C(20) & $90.53(15)$ \\
\hline Pt-C(20) & $2.102(5)$ & C(19)-Pt-C(20) & $87.15(17)$ \\
\hline Pt-I & $2.7830(5)$ & $\mathrm{N}(1)-\mathrm{Pt}-\mathrm{I}$ & $88.97(10)$ \\
\hline $\mathrm{N}(1)-\mathrm{C}(1)$ & $1.336(6)$ & N(3)-Pt-I & $92.59(10)$ \\
\hline $\mathrm{N}(1)-\mathrm{C}(9)$ & $1.375(6)$ & N(2)-Pt-I & $90.15(10)$ \\
\hline $\mathrm{N}(2)-\mathrm{C}(17)$ & $1.368(6)$ & C(19)-Pt-I & $92.18(13)$ \\
\hline $\mathrm{N}(2)-\mathrm{C}(8)$ & $1.370(6)$ & C(20)-Pt-I & $178.86(12)$ \\
\hline $\mathrm{N}(3)-\mathrm{C}(10)$ & $1.329(6)$ & $\mathrm{C}(1)-\mathrm{N}(1)-\mathrm{C}(9)$ & $119.9(4)$ \\
\hline $\mathrm{N}(3)-\mathrm{C}(18)$ & $1.380(6)$ & $\mathrm{C}(1)-\mathrm{N}(1)-\mathrm{Pt}$ & $127.9(3)$ \\
\hline $\mathrm{C}(1)-\mathrm{C}(2)$ & $1.398(7)$ & $\mathrm{C}(9)-\mathrm{N}(1)-\mathrm{Pt}$ & $112.2(3)$ \\
\hline $\mathrm{C}(1)-\mathrm{H}(1)$ & 0.9500 & $\mathrm{C}(17)-\mathrm{N}(2)-\mathrm{C}(8)$ & $131.9(4)$ \\
\hline$C(2)-C(3)$ & $1.369(7)$ & $\mathrm{C}(17)-\mathrm{N}(2)-\mathrm{Pt}$ & $114.0(3)$ \\
\hline $\mathrm{C}(2)-\mathrm{H}(2)$ & 0.9500 & $\mathrm{C}(8)-\mathrm{N}(2)-\mathrm{Pt}$ & 114.1(3) \\
\hline $\mathrm{C}(3)-\mathrm{C}(4)$ & $1.409(7)$ & $\mathrm{C}(10)-\mathrm{N}(3)-\mathrm{C}(18)$ & $119.8(4)$ \\
\hline $\mathrm{C}(3)-\mathrm{H}(3)$ & 0.9500 & $\mathrm{C}(10)-\mathrm{N}(3)-\mathrm{Pt}$ & $128.4(3)$ \\
\hline$C(4)-C(5)$ & $1.415(6)$ & $\mathrm{C}(18)-\mathrm{N}(3)-\mathrm{Pt}$ & $111.8(3)$ \\
\hline$C(4)-C(9)$ & $1.418(6)$ & $\mathrm{N}(1)-\mathrm{C}(1)-\mathrm{C}(2)$ & $122.4(4)$ \\
\hline$C(5)-C(6)$ & $1.361(7)$ & $\mathrm{N}(1)-\mathrm{C}(1)-\mathrm{H}(1)$ & 118.8 \\
\hline $\mathrm{C}(5)-\mathrm{H}(5)$ & 0.9500 & $\mathrm{C}(2)-\mathrm{C}(1)-\mathrm{H}(1)$ & 118.8 \\
\hline$C(6)-C(7)$ & $1.397(6)$ & $C(3)-C(2)-C(1)$ & $118.8(5)$ \\
\hline $\mathrm{C}(6)-\mathrm{H}(6)$ & 0.9500 & $\mathrm{C}(3)-\mathrm{C}(2)-\mathrm{H}(2)$ & 120.6 \\
\hline $\mathrm{C}(7)-\mathrm{C}(8)$ & $1.396(6)$ & $\mathrm{C}(1)-\mathrm{C}(2)-\mathrm{H}(2)$ & 120.6 \\
\hline $\mathrm{C}(7)-\mathrm{H}(7)$ & 0.9500 & $C(2)-C(3)-C(4)$ & $120.6(4)$ \\
\hline $\mathrm{C}(8)-\mathrm{C}(9)$ & $1.442(6)$ & $\mathrm{C}(2)-\mathrm{C}(3)-\mathrm{H}(3)$ & 119.7 \\
\hline$C(10)-C(11)$ & $1.406(7)$ & $\mathrm{C}(4)-\mathrm{C}(3)-\mathrm{H}(3)$ & 119.7 \\
\hline $\mathrm{C}(10)-\mathrm{H}(10)$ & 0.9500 & $C(3)-C(4)-C(5)$ & $123.0(4)$ \\
\hline C(11)-C(12) & $1.361(7)$ & $C(3)-C(4)-C(9)$ & $117.8(4)$ \\
\hline $\mathrm{C}(11)-\mathrm{H}(11)$ & 0.9500 & $C(5)-C(4)-C(9)$ & 119.2(4) \\
\hline$C(12)-C(13)$ & $1.409(6)$ & $C(6)-C(5)-C(4)$ & $118.9(4)$ \\
\hline $\mathrm{C}(12)-\mathrm{H}(12)$ & 0.9500 & $\mathrm{C}(6)-\mathrm{C}(5)-\mathrm{H}(5)$ & 120.6 \\
\hline C(13)-C(18) & $1.400(6)$ & $\mathrm{C}(4)-\mathrm{C}(5)-\mathrm{H}(5)$ & 120.6 \\
\hline$C(13)-C(14)$ & $1.417(6)$ & $C(5)-C(6)-C(7)$ & $122.8(4)$ \\
\hline$C(14)-C(15)$ & $1.354(7)$ & $\mathrm{C}(5)-\mathrm{C}(6)-\mathrm{H}(6)$ & 118.6 \\
\hline $\mathrm{C}(14)-\mathrm{H}(14)$ & 0.9500 & $\mathrm{C}(7)-\mathrm{C}(6)-\mathrm{H}(6)$ & 118.6 \\
\hline C(15)-C(16) & $1.412(7)$ & $C(8)-C(7)-C(6)$ & 121.3(4) \\
\hline $\mathrm{C}(15)-\mathrm{H}(15)$ & 0.9500 & $\mathrm{C}(8)-\mathrm{C}(7)-\mathrm{H}(7)$ & 119.4 \\
\hline$C(16)-C(17)$ & $1.380(6)$ & $\mathrm{C}(6)-\mathrm{C}(7)-\mathrm{H}(7)$ & 119.4 \\
\hline C(16)-H(16) & 0.9500 & $\mathrm{~N}(2)-\mathrm{C}(8)-\mathrm{C}(7)$ & $130.1(4)$ \\
\hline $\mathrm{C}(17)-\mathrm{C}(18)$ & $1.448(6)$ & $\mathrm{N}(2)-\mathrm{C}(8)-\mathrm{C}(9)$ & $113.4(4)$ \\
\hline C(19)-H(19A) & 0.9800 & $\mathrm{C}(7)-\mathrm{C}(8)-\mathrm{C}(9)$ & $116.4(4)$ \\
\hline C(19)-H(19B) & 0.9800 & $\mathrm{~N}(1)-\mathrm{C}(9)-\mathrm{C}(4)$ & $120.5(4)$ \\
\hline C(19)-H(19C) & 0.9800 & $\mathrm{~N}(1)-\mathrm{C}(9)-\mathrm{C}(8)$ & $118.2(4)$ \\
\hline $\mathrm{C}(20)-\mathrm{H}(20 \mathrm{~A})$ & 0.9800 & $\mathrm{C}(4)-\mathrm{C}(9)-\mathrm{C}(8)$ & 121.3(4) \\
\hline $\mathrm{C}(20)-\mathrm{H}(20 \mathrm{~B})$ & 0.9800 & $\mathrm{~N}(3)-\mathrm{C}(10)-\mathrm{C}(11)$ & $121.8(4)$ \\
\hline \multirow[t]{2}{*}{$\mathrm{C}(20)-\mathrm{H}(20 \mathrm{C})$} & 0.9800 & $\mathrm{~N}(3)-\mathrm{C}(10)-\mathrm{H}(10)$ & 119.1 \\
\hline & & $\mathrm{C}(11)-\mathrm{C}(10)-\mathrm{H}(10)$ & 119.1 \\
\hline N(1)-Pt-N(3) & $163.21(15)$ & $C(12)-C(11)-C(10)$ & $119.5(5)$ \\
\hline N(1)-Pt-N(2) & $81.48(15)$ & $\mathrm{C}(12)-\mathrm{C}(11)-\mathrm{H}(11)$ & 120.3 \\
\hline N(3)-Pt-N(2) & $81.80(15)$ & $\mathrm{C}(10)-\mathrm{C}(11)-\mathrm{H}(11)$ & 120.3 \\
\hline N(1)-Pt-C(19) & $99.05(17)$ & $C(11)-C(12)-C(13)$ & $119.8(5)$ \\
\hline N(3)-Pt-C(19) & $97.60(17)$ & $\mathrm{C}(11)-\mathrm{C}(12)-\mathrm{H}(12)$ & 120.1 \\
\hline
\end{tabular}




$\begin{array}{ll}\mathrm{C}(13)-\mathrm{C}(12)-\mathrm{H}(12) & 120.1 \\ \mathrm{C}(18)-\mathrm{C}(13)-\mathrm{C}(12) & 118.6(4) \\ \mathrm{C}(18)-\mathrm{C}(13)-\mathrm{C}(14) & 119.2(4) \\ \mathrm{C}(12)-\mathrm{C}(13)-\mathrm{C}(14) & 122.2(4) \\ \mathrm{C}(15)-\mathrm{C}(14)-\mathrm{C}(13) & 119.2(4) \\ \mathrm{C}(15)-\mathrm{C}(14)-\mathrm{H}(14) & 120.4 \\ \mathrm{C}(13)-\mathrm{C}(14)-\mathrm{H}(14) & 120.4 \\ \mathrm{C}(14)-\mathrm{C}(15)-\mathrm{C}(16) & 122.3(4) \\ \mathrm{C}(14)-\mathrm{C}(15)-\mathrm{H}(15) & 118.8 \\ \mathrm{C}(16)-\mathrm{C}(15)-\mathrm{H}(15) & 118.8 \\ \mathrm{C}(17)-\mathrm{C}(16)-\mathrm{C}(15) & 121.0(4) \\ \mathrm{C}(17)-\mathrm{C}(16)-\mathrm{H}(16) & 119.5 \\ \mathrm{C}(15)-\mathrm{C}(16)-\mathrm{H}(16) & 119.5 \\ \mathrm{~N}(2)-\mathrm{C}(17)-\mathrm{C}(16) & 129.4(4) \\ \mathrm{N}(2)-\mathrm{C}(17)-\mathrm{C}(18) & 113.7(4) \\ \mathrm{C}(16)-\mathrm{C}(17)-\mathrm{C}(18) & 116.8(4)\end{array}$

$\begin{array}{ll}\text { N(3)-C(18)-C(13) } & 120.5(4) \\ \text { N(3)-C(18)-C(17) } & 118.1(4) \\ \text { C(13)-C(18)-C(17) } & 121.3(4) \\ \text { Pt-C(19)-H(19A) } & 109.5 \\ \text { Pt-C(19)-H(19B) } & 109.5 \\ \text { H(19A)-C(19)-H(19B) } & 109.5 \\ \text { Pt-C(19)-H(19C) } & 109.5 \\ \text { H(19A)-C(19)-H(19C) } & 109.5 \\ \text { H(19B)-C(19)-H(19C) } & 109.5 \\ \text { Pt-C(20)-H(20A) } & 109.5 \\ \text { Pt-C(20)-H(20B) } & 109.5 \\ \text { H(20A)-C(20)-H(20B) } & 109.5 \\ \text { Pt-C(20)-H(20C) } & 109.5 \\ \text { H(20A)-C(20)-H(20C) } & 109.5 \\ \text { H(20B)-C(20)-H(20C) } & 109.5\end{array}$


Table 4. Anisotropic displacement parameters $\left(\AA^{2} \times 10^{3}\right)$ for 2 . The anisotropic displacement factor exponent takes the form: $-2 \pi^{2}\left[h^{2} a^{* 2} U^{11}+\ldots+2 h \mathrm{k} \mathrm{a}^{*} \mathrm{~b}^{*} \mathrm{U}^{12}\right]$

\begin{tabular}{lcccccc}
\hline & $\mathrm{U}^{11}$ & $\mathrm{U}^{22}$ & $\mathrm{U}^{33}$ & $\mathrm{U}^{23}$ & $\mathrm{U}^{13}$ & $\mathrm{U}^{12}$ \\
\hline $\mathrm{Pt}$ & $12(1)$ & $12(1)$ & $11(1)$ & $1(1)$ & $0(1)$ & $-1(1)$ \\
$\mathrm{I}$ & $22(1)$ & $15(1)$ & $17(1)$ & $1(1)$ & $0(1)$ & $2(1)$ \\
$\mathrm{N}(1)$ & $19(2)$ & $11(2)$ & $14(2)$ & $2(2)$ & $0(2)$ & $-1(2)$ \\
$\mathrm{N}(2)$ & $15(2)$ & $12(2)$ & $13(2)$ & $1(2)$ & $1(2)$ & $-1(2)$ \\
$\mathrm{N}(3)$ & $17(2)$ & $12(2)$ & $12(2)$ & $1(2)$ & $-2(2)$ & $0(2)$ \\
$\mathrm{C}(1)$ & $16(2)$ & $14(2)$ & $21(2)$ & $1(2)$ & $1(2)$ & $-5(2)$ \\
$\mathrm{C}(2)$ & $26(3)$ & $19(3)$ & $19(2)$ & $4(2)$ & $3(2)$ & $1(2)$ \\
$\mathrm{C}(3)$ & $26(3)$ & $15(2)$ & $13(2)$ & $3(2)$ & $3(2)$ & $7(2)$ \\
$\mathrm{C}(4)$ & $20(2)$ & $8(2)$ & $17(2)$ & $2(2)$ & $-1(2)$ & $2(2)$ \\
$\mathrm{C}(5)$ & $24(2)$ & $16(2)$ & $12(2)$ & $-3(2)$ & $-5(2)$ & $5(2)$ \\
$\mathrm{C}(6)$ & $17(2)$ & $16(2)$ & $24(3)$ & $-2(2)$ & $-9(2)$ & $3(2)$ \\
$\mathrm{C}(7)$ & $16(2)$ & $17(2)$ & $18(2)$ & $0(2)$ & $2(2)$ & $0(2)$ \\
$\mathrm{C}(8)$ & $17(2)$ & $10(2)$ & $13(2)$ & $-1(2)$ & $1(2)$ & $4(2)$ \\
$\mathrm{C}(9)$ & $17(2)$ & $9(2)$ & $13(2)$ & $1(2)$ & $-3(2)$ & $2(2)$ \\
$\mathrm{C}(10)$ & $19(2)$ & $18(2)$ & $18(3)$ & $-1(2)$ & $-4(2)$ & $1(2)$ \\
$\mathrm{C}(11)$ & $26(3)$ & $19(3)$ & $16(2)$ & $-4(2)$ & $-8(2)$ & $3(2)$ \\
$\mathrm{C}(12)$ & $28(3)$ & $19(2)$ & $13(2)$ & $0(2)$ & $2(2)$ & $8(2)$ \\
$\mathrm{C}(13)$ & $21(2)$ & $14(2)$ & $14(2)$ & $0(2)$ & $1(2)$ & $4(2)$ \\
$\mathrm{C}(14)$ & $20(2)$ & $20(3)$ & $16(2)$ & $4(2)$ & $10(2)$ & $4(2)$ \\
$\mathrm{C}(15)$ & $14(2)$ & $24(3)$ & $22(3)$ & $6(2)$ & $0(2)$ & $-1(2)$ \\
$\mathrm{C}(16)$ & $13(2)$ & $16(2)$ & $23(3)$ & $-4(2)$ & $-2(2)$ & $2(2)$ \\
$\mathrm{C}(17)$ & $16(2)$ & $11(2)$ & $15(2)$ & $-1(2)$ & $2(2)$ & $5(2)$ \\
$\mathrm{C}(18)$ & $15(2)$ & $8(2)$ & $16(2)$ & $1(2)$ & $-3(2)$ & $4(2)$ \\
$\mathrm{C}(19)$ & $18(2)$ & $15(2)$ & $18(2)$ & $0(2)$ & $3(2)$ & $-4(2)$ \\
$\mathrm{C}(20)$ & $13(2)$ & $31(3)$ & $8(2)$ & $7(2)$ & $-5(2)$ & $-14(2)$ \\
& & & & & & \\
\hline
\end{tabular}


Table 5. Hydrogen coordinates ( $\left.\times 10^{4}\right)$ and isotropic displacement parameters $\left(\AA^{2} \times 10^{3}\right)$ for 2 .

\begin{tabular}{lrrrr}
\hline & $\mathrm{x}$ & $\mathrm{y}$ & $\mathrm{z}$ & $\mathrm{U}(\mathrm{eq})$ \\
\hline $\mathrm{H}(1)$ & 4089 & 1893 & 730 & 20 \\
$\mathrm{H}(2)$ & 3754 & 1760 & -882 & 25 \\
$\mathrm{H}(3)$ & 1212 & 1300 & -1494 & 22 \\
$\mathrm{H}(5)$ & -1779 & 848 & -1228 & 21 \\
$\mathrm{H}(6)$ & -3838 & 549 & -179 & 23 \\
$\mathrm{H}(7)$ & -3383 & 621 & 1414 & 20 \\
$\mathrm{H}(10)$ & 3076 & 2169 & 4457 & 22 \\
$\mathrm{H}(11)$ & 2090 & 1986 & 5973 & 25 \\
$\mathrm{H}(12)$ & -398 & 1269 & 6207 & 24 \\
$\mathrm{H}(14)$ & -2866 & 475 & 5527 & 22 \\
$\mathrm{H}(15)$ & -4174 & -89 & 4237 & 24 \\
$\mathrm{H}(16)$ & -3182 & 148 & 2737 & 21 \\
$\mathrm{H}(19 \mathrm{~A})$ & 4678 & 1970 & 3240 & 26 \\
$\mathrm{H}(19 \mathrm{~B})$ & 4133 & 2799 & 2653 & 26 \\
$\mathrm{H}(19 \mathrm{C})$ & 4918 & 1987 & 2137 & 26 \\
$\mathrm{H}(20 \mathrm{~A})$ & 2064 & 10 & 2727 & 26 \\
$\mathrm{H}(20 \mathrm{~B})$ & 3727 & 449 & 3129 & 26 \\
$\mathrm{H}(20 \mathrm{C})$ & 3509 & 289 & 2034 & 26 \\
& & & & \\
\hline
\end{tabular}


Table 6. Crystal data and structure refinement for 3.

\begin{tabular}{|c|c|c|}
\hline Name & \multicolumn{2}{|l|}{ (fac-BQA)PtMe $2 \mathrm{I}$} \\
\hline Empirical formula & \multicolumn{2}{|l|}{$\mathrm{C}_{20} \mathrm{H}_{18} \mathrm{IN}{ }_{3} \mathrm{Pt}$} \\
\hline Formula weight & \multicolumn{2}{|l|}{622.36} \\
\hline Temperature & \multicolumn{2}{|l|}{$98(2) \mathrm{K}$} \\
\hline Wavelength & \multicolumn{2}{|l|}{$0.71073 \AA$} \\
\hline Crystal habit & \multicolumn{2}{|l|}{ Rough block } \\
\hline Crystal color & \multicolumn{2}{|l|}{ Red } \\
\hline Crystal system & \multicolumn{2}{|l|}{ Monoclinic } \\
\hline Space group & \multicolumn{2}{|l|}{ P 21/n (\#14) } \\
\hline \multirow[t]{3}{*}{ Unit cell dimensions } & $\mathrm{a}=7.9108(8) \AA$ & $\alpha=90^{\circ}$ \\
\hline & $\mathrm{b}=15.9623(16) \AA$ & $\beta=90.771(2)^{\circ}$ \\
\hline & $\mathrm{c}=14.5739(15) \AA$ & $\gamma=90^{\circ}$ \\
\hline Volume & \multicolumn{2}{|l|}{$1840.1(3) \AA^{3}$} \\
\hline & \multirow{2}{*}{\multicolumn{2}{|c|}{$\begin{array}{l}4 \\
2246 \mathrm{Mg} / \mathrm{m}^{3}\end{array}$}} \\
\hline Density (calculated) & & \\
\hline Absorption coefficient & \multicolumn{2}{|l|}{$9.310 \mathrm{~mm}^{-1}$} \\
\hline $\mathrm{F}(000)$ & \multicolumn{2}{|c|}{1160} \\
\hline Crystal size & \multicolumn{2}{|c|}{$0.15 \times 0.15 \times 0.11 \mathrm{~mm}^{3}$} \\
\hline Theta range for data collection & \multicolumn{2}{|c|}{1.89 to $28.33^{\circ}$} \\
\hline Index ranges & \multicolumn{2}{|c|}{$-10<=\mathrm{h}<=10,-20<=\mathrm{k}<=20,-19<=1<=19$} \\
\hline Reflections collected & \multicolumn{2}{|c|}{26315} \\
\hline Independent reflections & \multicolumn{2}{|c|}{$4305[\mathrm{R}(\mathrm{int})=0.0386]$} \\
\hline Completeness to theta $=28.33^{\circ}$ & \multicolumn{2}{|c|}{$93.8 \%$} \\
\hline Absorption correction & \multicolumn{2}{|c|}{ Semi-empirical from equivalents } \\
\hline Refinement method & \multirow{2}{*}{\multicolumn{2}{|c|}{ Full-matrix least-squares on $\mathrm{F}^{2}$}} \\
\hline Data / restraints / parameters & & \\
\hline Goodness-of-fit on $\mathrm{F}^{2}$ & \multicolumn{2}{|l|}{1.087} \\
\hline Final $R$ indices $[\mathrm{I}>2 \operatorname{sigma}(\mathrm{I})]$ & \multicolumn{2}{|c|}{$\mathrm{R} 1=0.0202, \mathrm{wR} 2=0.0466$} \\
\hline $\mathrm{R}$ indices (all data) & \multicolumn{2}{|c|}{$\mathrm{R} 1=0.0234, \mathrm{wR} 2=0.0474$} \\
\hline Largest diff. peak and hole & \multicolumn{2}{|c|}{1.331 and $-0.830 \mathrm{e} \cdot \AA^{-3}$} \\
\hline
\end{tabular}

\section{Refinement Details}

Refinement of $\mathrm{F}^{2}$ against all reflections. The weighted $\mathrm{R}$-factor $\mathrm{wR}$ and goodness of fit $\mathrm{S}$ are based on $\mathrm{F}^{2}$, conventional R-factors $\mathrm{R}$ are based on $\mathrm{F}$, with $\mathrm{F}$ set to zero for negative $\mathrm{F}^{2}$. The threshold expression of $\mathrm{F}^{2}>$ $2 \operatorname{sigma}\left(\mathrm{F}^{2}\right)$ is used only for calculating $\mathrm{R}$-factors $(\mathrm{gt})$ etc. and is not relevant to the choice of reflections for refinement. R-factors based on $\mathrm{F}^{2}$ are statistically about twice as large as those based on $\mathrm{F}$, and $\mathrm{R}$ - factors based on all data will be even larger.

All esds (except the esd in the dihedral angle between two 1.s. planes) are estimated using the full covariance matrix. The cell esds are taken into account individually in the estimation of esds in distances, angles and torsion angles; correlations between esds in cell parameters are only used when they are defined by crystal symmetry. An approximate (isotropic) treatment of cell esds is used for estimating esds involving l.s. planes. 
Table 7. Atomic coordinates ( $\left.\mathrm{x} 10^{4}\right)$ and equivalent isotropic displacement parameters $\left(\AA^{2} \times 10^{3}\right)$ for 3. $U(e q)$ is defined as one third of the trace of the orthogonalized $U^{i j}$ tensor.

\begin{tabular}{lrrrr}
\hline & $\mathrm{x}$ & $\mathrm{y}$ & $\mathrm{z}$ & $\mathrm{U}(\mathrm{eq})$ \\
\hline $\mathrm{Pt}$ & $1425(1)$ & $3738(1)$ & $7439(1)$ & $10(1)$ \\
$\mathrm{I}$ & $28(1)$ & $4497(1)$ & $8839(1)$ & $17(1)$ \\
$\mathrm{N}(1)$ & $1225(3)$ & $4737(2)$ & $6468(2)$ & $12(1)$ \\
$\mathrm{N}(2)$ & $2416(3)$ & $3148(2)$ & $6330(2)$ & $12(1)$ \\
$\mathrm{N}(3)$ & $-756(3)$ & $3044(2)$ & $6990(2)$ & $13(1)$ \\
$\mathrm{C}(1)$ & $578(4)$ & $5490(2)$ & $6562(2)$ & $16(1)$ \\
$\mathrm{C}(2)$ & $770(5)$ & $6121(2)$ & $5900(3)$ & $20(1)$ \\
$\mathrm{C}(3)$ & $1698(4)$ & $5957(2)$ & $5139(2)$ & $19(1)$ \\
$\mathrm{C}(4)$ & $2390(4)$ & $5155(2)$ & $5006(2)$ & $17(1)$ \\
$\mathrm{C}(5)$ & $3291(4)$ & $4915(2)$ & $4218(2)$ & $22(1)$ \\
$\mathrm{C}(6)$ & $3902(4)$ & $4121(3)$ & $4142(2)$ & $23(1)$ \\
$\mathrm{C}(7)$ & $3666(4)$ & $3521(2)$ & $4838(2)$ & $18(1)$ \\
$\mathrm{C}(8)$ & $2780(4)$ & $3717(2)$ & $5620(2)$ & $13(1)$ \\
$\mathrm{C}(9)$ & $2138(4)$ & $4553(2)$ & $5700(2)$ & $14(1)$ \\
$\mathrm{C}(10)$ & $-2341(4)$ & $3066(2)$ & $7272(2)$ & $18(1)$ \\
$\mathrm{C}(11)$ & $-3572(4)$ & $2497(2)$ & $6976(3)$ & $23(1)$ \\
$\mathrm{C}(12)$ & $-3124(4)$ & $1866(2)$ & $6397(3)$ & $22(1)$ \\
$\mathrm{C}(13)$ & $-1436(4)$ & $1810(2)$ & $6082(2)$ & $17(1)$ \\
$\mathrm{C}(14)$ & $-863(5)$ & $1181(2)$ & $5474(3)$ & $22(1)$ \\
$\mathrm{C}(15)$ & $778(5)$ & $1185(2)$ & $5204(3)$ & $22(1)$ \\
$\mathrm{C}(16)$ & $1915(4)$ & $1813(2)$ & $5493(2)$ & $17(1)$ \\
$\mathrm{C}(17)$ & $1406(4)$ & $2453(2)$ & $6060(2)$ & $13(1)$ \\
$\mathrm{C}(18)$ & $-291(4)$ & $2425(2)$ & $6390(2)$ & $13(1)$ \\
$\mathrm{C}(19)$ & $3709(4)$ & $4265(2)$ & $7791(2)$ & $17(1)$ \\
$\mathrm{C}(20)$ & $1930(4)$ & $2733(2)$ & $8270(2)$ & $17(1)$ \\
& & & & \\
\hline
\end{tabular}


Table 8. Bond lengths $[\AA]$ and angles $\left[{ }^{\circ}\right]$ for 3 .

\begin{tabular}{|c|c|c|c|}
\hline $\mathrm{Pt}-\mathrm{N}(2)$ & $2.036(3)$ & C(19)-Pt-N(1) & $85.19(12)$ \\
\hline Pt-C(20) & $2.046(3)$ & $\mathrm{N}(2)-\mathrm{Pt}-\mathrm{N}(3)$ & $80.54(10)$ \\
\hline Pt-C(19) & $2.052(3)$ & C(20)-Pt-N(3) & $85.74(12)$ \\
\hline Pt-N(1) & $2.137(3)$ & C(19)-Pt-N(3) & $171.87(12)$ \\
\hline Pt-N(3) & $2.146(3)$ & N(1)-Pt-N(3) & $97.51(10)$ \\
\hline Pt-I & $2.6299(3)$ & N(2)-Pt-I & $177.72(7)$ \\
\hline $\mathrm{N}(1)-\mathrm{C}(1)$ & $1.315(4)$ & C(20)-Pt-I & 89.02(10) \\
\hline $\mathrm{N}(1)-\mathrm{C}(9)$ & $1.372(4)$ & C(19)-Pt-I & $89.70(10)$ \\
\hline $\mathrm{N}(2)-\mathrm{C}(8)$ & $1.409(4)$ & $\mathrm{N}(1)-\mathrm{Pt}-\mathrm{I}$ & $98.16(7)$ \\
\hline $\mathrm{N}(2)-\mathrm{C}(17)$ & $1.421(4)$ & N(3)-Pt-I & $97.47(7)$ \\
\hline $\mathrm{N}(3)-\mathrm{C}(10)$ & $1.325(4)$ & $\mathrm{C}(1)-\mathrm{N}(1)-\mathrm{C}(9)$ & $119.4(3)$ \\
\hline $\mathrm{N}(3)-\mathrm{C}(18)$ & $1.373(4)$ & $\mathrm{C}(1)-\mathrm{N}(1)-\mathrm{Pt}$ & $129.7(2)$ \\
\hline$C(1)-C(2)$ & $1.403(5)$ & $\mathrm{C}(9)-\mathrm{N}(1)-\mathrm{Pt}$ & $110.2(2)$ \\
\hline $\mathrm{C}(1)-\mathrm{H}(1)$ & 0.9500 & $\mathrm{C}(8)-\mathrm{N}(2)-\mathrm{C}(17)$ & $114.8(3)$ \\
\hline $\mathrm{C}(2)-\mathrm{C}(3)$ & $1.364(5)$ & $\mathrm{C}(8)-\mathrm{N}(2)-\mathrm{Pt}$ & $111.7(2)$ \\
\hline $\mathrm{C}(2)-\mathrm{H}(2)$ & 0.9500 & $\mathrm{C}(17)-\mathrm{N}(2)-\mathrm{Pt}$ & $111.11(19)$ \\
\hline$C(3)-C(4)$ & $1.406(5)$ & $\mathrm{C}(10)-\mathrm{N}(3)-\mathrm{C}(18)$ & 118.7(3) \\
\hline $\mathrm{C}(3)-\mathrm{H}(3)$ & 0.9500 & $\mathrm{C}(10)-\mathrm{N}(3)-\mathrm{Pt}$ & $130.6(2)$ \\
\hline $\mathrm{C}(4)-\mathrm{C}(9)$ & $1.411(5)$ & $\mathrm{C}(18)-\mathrm{N}(3)-\mathrm{Pt}$ & $110.2(2)$ \\
\hline $\mathrm{C}(4)-\mathrm{C}(5)$ & $1.414(5)$ & $\mathrm{N}(1)-\mathrm{C}(1)-\mathrm{C}(2)$ & $122.6(3)$ \\
\hline$C(5)-C(6)$ & $1.361(6)$ & $\mathrm{N}(1)-\mathrm{C}(1)-\mathrm{H}(1)$ & 118.7 \\
\hline $\mathrm{C}(5)-\mathrm{H}(5)$ & 0.9500 & $\mathrm{C}(2)-\mathrm{C}(1)-\mathrm{H}(1)$ & 118.7 \\
\hline$C(6)-C(7)$ & $1.409(5)$ & $C(3)-C(2)-C(1)$ & $119.0(3)$ \\
\hline $\mathrm{C}(6)-\mathrm{H}(6)$ & 0.9500 & $\mathrm{C}(3)-\mathrm{C}(2)-\mathrm{H}(2)$ & 120.5 \\
\hline$C(7)-C(8)$ & $1.382(5)$ & $\mathrm{C}(1)-\mathrm{C}(2)-\mathrm{H}(2)$ & 120.5 \\
\hline $\mathrm{C}(7)-\mathrm{H}(7)$ & 0.9500 & $\mathrm{C}(2)-\mathrm{C}(3)-\mathrm{C}(4)$ & 120.1(3) \\
\hline $\mathrm{C}(8)-\mathrm{C}(9)$ & $1.433(5)$ & $\mathrm{C}(2)-\mathrm{C}(3)-\mathrm{H}(3)$ & 119.9 \\
\hline$C(10)-C(11)$ & $1.396(5)$ & $\mathrm{C}(4)-\mathrm{C}(3)-\mathrm{H}(3)$ & 119.9 \\
\hline $\mathrm{C}(10)-\mathrm{H}(10)$ & 0.9500 & $\mathrm{C}(3)-\mathrm{C}(4)-\mathrm{C}(9)$ & $117.5(3)$ \\
\hline C(11)-C(12) & $1.363(5)$ & $C(3)-C(4)-C(5)$ & 124.1(3) \\
\hline C(11)-H(11) & 0.9500 & $C(9)-C(4)-C(5)$ & 118.4(3) \\
\hline$C(12)-C(13)$ & $1.420(5)$ & $C(6)-C(5)-C(4)$ & $120.1(3)$ \\
\hline $\mathrm{C}(12)-\mathrm{H}(12)$ & 0.9500 & $\mathrm{C}(6)-\mathrm{C}(5)-\mathrm{H}(5)$ & 119.9 \\
\hline C(13)-C(18) & $1.404(5)$ & $\mathrm{C}(4)-\mathrm{C}(5)-\mathrm{H}(5)$ & 119.9 \\
\hline C(13)-C(14) & $1.419(5)$ & $C(5)-C(6)-C(7)$ & $121.6(3)$ \\
\hline$C(14)-C(15)$ & $1.361(5)$ & $\mathrm{C}(5)-\mathrm{C}(6)-\mathrm{H}(6)$ & 119.2 \\
\hline $\mathrm{C}(14)-\mathrm{H}(14)$ & 0.9500 & $\mathrm{C}(7)-\mathrm{C}(6)-\mathrm{H}(6)$ & 119.2 \\
\hline$C(15)-C(16)$ & $1.408(5)$ & $C(8)-C(7)-C(6)$ & $120.9(3)$ \\
\hline $\mathrm{C}(15)-\mathrm{H}(15)$ & 0.9500 & $\mathrm{C}(8)-\mathrm{C}(7)-\mathrm{H}(7)$ & 119.6 \\
\hline$C(16)-C(17)$ & $1.377(4)$ & $\mathrm{C}(6)-\mathrm{C}(7)-\mathrm{H}(7)$ & 119.6 \\
\hline $\mathrm{C}(16)-\mathrm{H}(16)$ & 0.9500 & $\mathrm{C}(7)-\mathrm{C}(8)-\mathrm{N}(2)$ & $124.8(3)$ \\
\hline C(17)-C(18) & $1.433(4)$ & $\mathrm{C}(7)-\mathrm{C}(8)-\mathrm{C}(9)$ & $117.5(3)$ \\
\hline $\mathrm{C}(19)-\mathrm{H}(19 \mathrm{~A})$ & 0.9800 & $\mathrm{~N}(2)-\mathrm{C}(8)-\mathrm{C}(9)$ & $117.6(3)$ \\
\hline C(19)-H(19B) & 0.9800 & $\mathrm{~N}(1)-\mathrm{C}(9)-\mathrm{C}(4)$ & $121.3(3)$ \\
\hline $\mathrm{C}(19)-\mathrm{H}(19 \mathrm{C})$ & 0.9800 & $\mathrm{~N}(1)-\mathrm{C}(9)-\mathrm{C}(8)$ & $117.2(3)$ \\
\hline $\mathrm{C}(20)-\mathrm{H}(20 \mathrm{~A})$ & 0.9800 & $\mathrm{C}(4)-\mathrm{C}(9)-\mathrm{C}(8)$ & $121.5(3)$ \\
\hline $\mathrm{C}(20)-\mathrm{H}(20 \mathrm{~B})$ & 0.9800 & $\mathrm{~N}(3)-\mathrm{C}(10)-\mathrm{C}(11)$ & $123.0(3)$ \\
\hline \multirow[t]{2}{*}{$\mathrm{C}(20)-\mathrm{H}(20 \mathrm{C})$} & 0.9800 & $\mathrm{~N}(3)-\mathrm{C}(10)-\mathrm{H}(10)$ & 118.5 \\
\hline & & $\mathrm{C}(11)-\mathrm{C}(10)-\mathrm{H}(10)$ & 118.5 \\
\hline N(2)-Pt-C(20) & $91.93(12)$ & $C(12)-C(11)-C(10)$ & 119.1(3) \\
\hline N(2)-Pt-C(19) & $92.36(12)$ & $\mathrm{C}(12)-\mathrm{C}(11)-\mathrm{H}(11)$ & 120.4 \\
\hline $\mathrm{C}(20)-\mathrm{Pt}-\mathrm{C}(19)$ & $90.58(14)$ & $\mathrm{C}(10)-\mathrm{C}(11)-\mathrm{H}(11)$ & 120.4 \\
\hline N(2)-Pt-N(1) & $81.06(10)$ & $\mathrm{C}(11)-\mathrm{C}(12)-\mathrm{C}(13)$ & $120.0(3)$ \\
\hline $\mathrm{C}(20)-\mathrm{Pt}-\mathrm{N}(1)$ & $171.63(12)$ & $\mathrm{C}(11)-\mathrm{C}(12)-\mathrm{H}(12)$ & 120.0 \\
\hline
\end{tabular}




$\begin{array}{ll}\text { C(13)-C(12)-H(12) } & 120.0 \\ \text { C(18)-C(13)-C(14) } & 118.9(3) \\ \text { C(18)-C(13)-C(12) } & 117.3(3) \\ \text { C(14)-C(13)-C(12) } & 123.8(3) \\ \text { C(15)-C(14)-C(13) } & 119.5(3) \\ \text { C(15)-C(14)-H(14) } & 120.3 \\ \text { C(13)-C(14)-H(14) } & 120.3 \\ \text { C(14)-C(15)-C(16) } & 121.6(3) \\ \text { C(14)-C(15)-H(15) } & 119.2 \\ \text { C(16)-C(15)-H(15) } & 119.2 \\ \text { C(17)-C(16)-C(15) } & 121.1(3) \\ \text { C(17)-C(16)-H(16) } & 119.4 \\ \text { C(15)-C(16)-H(16) } & 119.4 \\ \text { C(16)-C(17)-N(2) } & 125.3(3) \\ \text { C(16)-C(17)-C(18) } & 117.5(3) \\ \text { N(2)-C(17)-C(18) } & 117.2(3) \\ \text { N(3)-C(18)-C(13) } & 121.9(3) \\ \text { N(3)-C(18)-C(17) } & 116.9(3) \\ \text { C(13)-C(18)-C(17) } & 121.2(3) \\ \text { Pt-C(19)-H(19A) } & 109.5 \\ \text { Pt-C(19)-H(19B) } & 109.5 \\ \text { H(19A)-C(19)-H(19B) } & 109.5 \\ \text { Pt-C(19)-H(19C) } & 109.5 \\ \text { H(19A)-C(19)-H(19C) } & 109.5 \\ \text { H(19B)-C(19)-H(19C) } & 109.5 \\ \text { Pt-C(20)-H(20A) } & 109.5 \\ \text { Pt-C(20)-H(20B) } & 109.5 \\ \text { H(20A)-C(20)-H(20B) } & 109.5 \\ \text { Pt-C(20)-H(20C) } & 109.5 \\ \text { H(20A)-C(20)-H(20C) } & 109.5 \\ \text { H(20B)-C(20)-H(20C) } & 109.5 \\ & \end{array}$


Table 9. Anisotropic displacement parameters $\left(\AA^{2} \times 10^{3}\right)$ for 3. The anisotropic displacement factor exponent takes the form: $-2 \pi^{2}\left[\mathrm{~h}^{2} \mathrm{a}^{* 2} \mathrm{U}^{11}+\ldots+2 \mathrm{~h} \mathrm{k} \mathrm{a}^{*} \mathrm{~b}^{*} \mathrm{U}^{12}\right]$

\begin{tabular}{lcccccc}
\hline & $\mathrm{U}^{11}$ & $\mathrm{U}^{22}$ & $\mathrm{U}^{33}$ & $\mathrm{U}^{23}$ & $\mathrm{U}^{13}$ & $\mathrm{U}^{12}$ \\
\hline $\mathrm{Pt}$ & $10(1)$ & $11(1)$ & $9(1)$ & $0(1)$ & $2(1)$ & $1(1)$ \\
$\mathrm{I}$ & $20(1)$ & $19(1)$ & $13(1)$ & $-3(1)$ & $5(1)$ & $3(1)$ \\
$\mathrm{N}(1)$ & $10(1)$ & $13(1)$ & $14(1)$ & $-2(1)$ & $-2(1)$ & $0(1)$ \\
$\mathrm{N}(2)$ & $10(1)$ & $14(1)$ & $11(1)$ & $0(1)$ & $2(1)$ & $0(1)$ \\
$\mathrm{N}(3)$ & $12(1)$ & $13(1)$ & $14(1)$ & $2(1)$ & $2(1)$ & $2(1)$ \\
$\mathrm{C}(1)$ & $16(2)$ & $15(2)$ & $18(2)$ & $-2(1)$ & $-4(1)$ & $-3(1)$ \\
$\mathrm{C}(2)$ & $21(2)$ & $13(2)$ & $24(2)$ & $2(1)$ & $-8(1)$ & $-3(1)$ \\
$\mathrm{C}(3)$ & $20(2)$ & $16(2)$ & $20(2)$ & $7(1)$ & $-8(1)$ & $-9(1)$ \\
$\mathrm{C}(4)$ & $12(2)$ & $21(2)$ & $17(2)$ & $4(1)$ & $-5(1)$ & $-7(1)$ \\
$\mathrm{C}(5)$ & $19(2)$ & $29(2)$ & $18(2)$ & $9(2)$ & $0(1)$ & $-8(2)$ \\
$\mathrm{C}(6)$ & $17(2)$ & $38(2)$ & $14(2)$ & $4(2)$ & $5(1)$ & $-2(2)$ \\
$\mathrm{C}(7)$ & $15(2)$ & $23(2)$ & $16(2)$ & $0(1)$ & $5(1)$ & $3(1)$ \\
$\mathrm{C}(8)$ & $12(2)$ & $18(2)$ & $10(2)$ & $1(1)$ & $0(1)$ & $-3(1)$ \\
$\mathrm{C}(9)$ & $11(2)$ & $17(2)$ & $14(2)$ & $-1(1)$ & $-1(1)$ & $-4(1)$ \\
$\mathrm{C}(10)$ & $17(2)$ & $16(2)$ & $22(2)$ & $2(1)$ & $5(1)$ & $1(1)$ \\
$\mathrm{C}(11)$ & $12(2)$ & $22(2)$ & $34(2)$ & $4(2)$ & $5(1)$ & $0(1)$ \\
$\mathrm{C}(12)$ & $15(2)$ & $18(2)$ & $31(2)$ & $3(2)$ & $-3(1)$ & $-4(1)$ \\
$\mathrm{C}(13)$ & $18(2)$ & $12(2)$ & $20(2)$ & $3(1)$ & $-2(1)$ & $1(1)$ \\
$\mathrm{C}(14)$ & $26(2)$ & $13(2)$ & $26(2)$ & $-3(1)$ & $-5(2)$ & $-1(1)$ \\
$\mathrm{C}(15)$ & $28(2)$ & $17(2)$ & $21(2)$ & $-6(1)$ & $-1(2)$ & $5(1)$ \\
$\mathrm{C}(16)$ & $16(2)$ & $17(2)$ & $18(2)$ & $-3(1)$ & $2(1)$ & $2(1)$ \\
$\mathrm{C}(17)$ & $13(2)$ & $13(2)$ & $13(2)$ & $3(1)$ & $1(1)$ & $2(1)$ \\
$\mathrm{C}(18)$ & $13(2)$ & $12(2)$ & $13(2)$ & $3(1)$ & $0(1)$ & $1(1)$ \\
$\mathrm{C}(19)$ & $12(2)$ & $24(2)$ & $15(2)$ & $-2(1)$ & $0(1)$ & $-2(1)$ \\
$\mathrm{C}(20)$ & $19(2)$ & $17(2)$ & $16(2)$ & $4(1)$ & $2(1)$ & $4(1)$ \\
& & & & & & \\
\hline
\end{tabular}


Table 10. Hydrogen coordinates $\left(\times 10^{4}\right)$ and isotropic displacement parameters $\left(\AA^{2} \times 10^{3}\right)$ for 3.

\begin{tabular}{lrrrl}
\hline & $\mathrm{x}$ & $\mathrm{y}$ & $\mathrm{z}$ & $\mathrm{U}(\mathrm{eq})$ \\
\hline $\mathrm{H}(1)$ & & & & \\
$\mathrm{H}(2)$ & -45 & 5612 & 7098 & 20 \\
$\mathrm{H}(3)$ & 261 & 6654 & 5982 & 23 \\
$\mathrm{H}(5)$ & 1878 & 6385 & 4698 & 23 \\
$\mathrm{H}(6)$ & 3469 & 5308 & 3739 & 26 \\
$\mathrm{H}(7)$ & 4502 & 3968 & 3607 & 27 \\
$\mathrm{H}(10)$ & 4122 & 2974 & 4769 & 22 \\
$\mathrm{H}(11)$ & -2654 & 3489 & 7696 & 22 \\
$\mathrm{H}(12)$ & -4706 & 2549 & 7174 & 27 \\
$\mathrm{H}(14)$ & -3940 & 1464 & 6205 & 26 \\
$\mathrm{H}(15)$ & -1617 & 760 & 5257 & 26 \\
$\mathrm{H}(16)$ & 1165 & 753 & 4810 & 27 \\
$\mathrm{H}(19 \mathrm{~A})$ & 3053 & 1795 & 5294 & 21 \\
$\mathrm{H}(19 \mathrm{~B})$ & 4508 & 4181 & 7291 & 26 \\
$\mathrm{H}(19 \mathrm{C})$ & 4150 & 4000 & 8351 & 26 \\
$\mathrm{H}(20 \mathrm{~A})$ & 3558 & 4867 & 7898 & 26 \\
$\mathrm{H}(20 \mathrm{~B})$ & 2446 & 2285 & 7909 & 26 \\
$\mathrm{H}(20 \mathrm{C})$ & 875 & 2529 & 8536 & 26 \\
& 2711 & 2903 & 8764 & 26 \\
\hline
\end{tabular}


Table 11. Crystal data and structure refinement for 4.

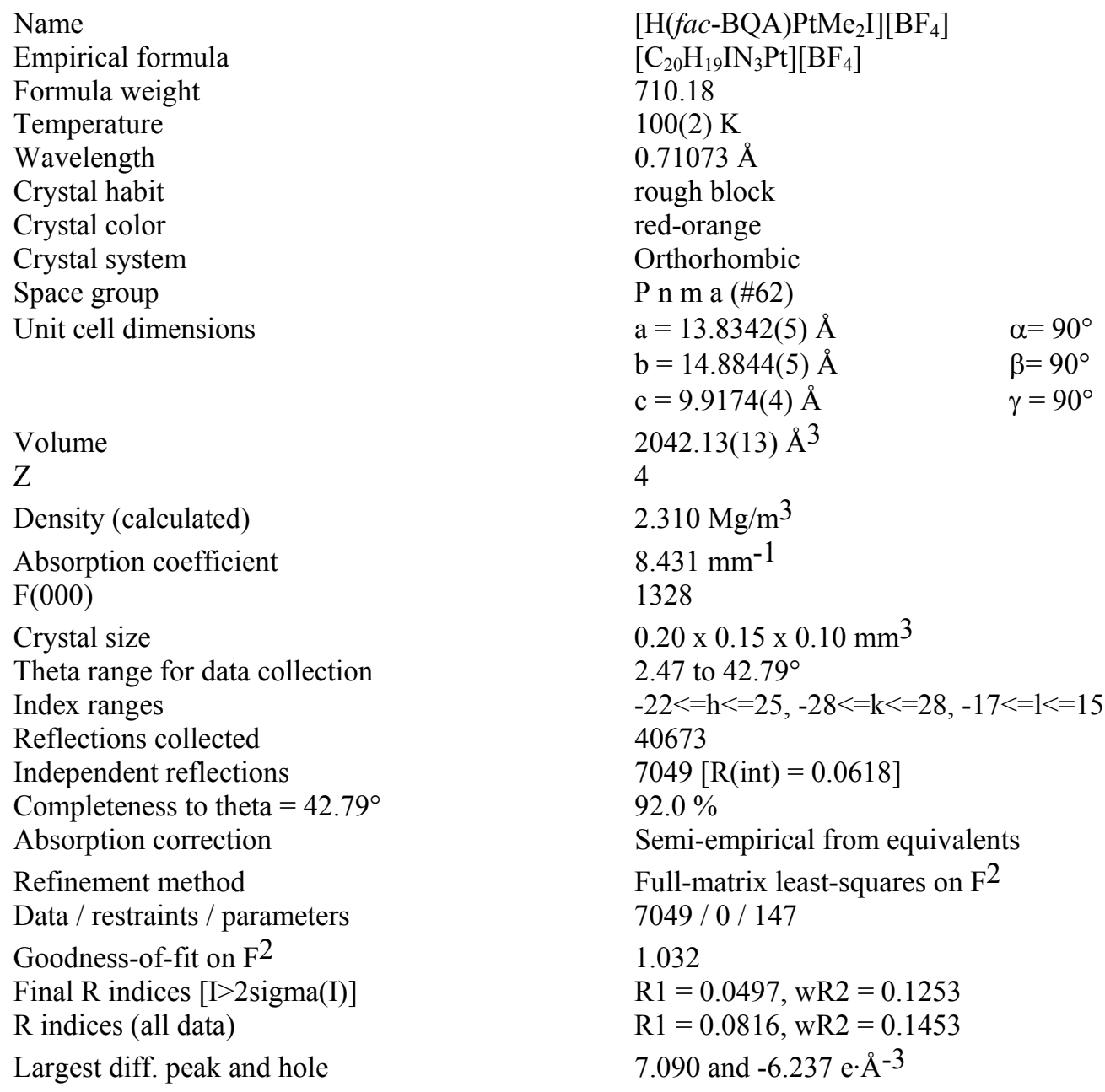

\section{Refinement Details}

Refinement of $\mathrm{F}^{2}$ against all reflections. The weighted $\mathrm{R}$-factor $\mathrm{wR}$ and goodness of fit $\mathrm{S}$ are based on $\mathrm{F}^{2}$, conventional R-factors $\mathrm{R}$ are based on $\mathrm{F}$, with $\mathrm{F}$ set to zero for negative $\mathrm{F}^{2}$. The threshold expression of $\mathrm{F}^{2}>$ $2 \operatorname{sigma}\left(\mathrm{F}^{2}\right)$ is used only for calculating R-factors(gt) etc. and is not relevant to the choice of reflections for refinement. R-factors based on $\mathrm{F}^{2}$ are statistically about twice as large as those based on $\mathrm{F}$, and $\mathrm{R}$ - factors based on all data will be even larger.

All esds (except the esd in the dihedral angle between two 1.s. planes) are estimated using the full covariance matrix. The cell esds are taken into account individually in the estimation of esds in distances, angles and torsion angles; correlations between esds in cell parameters are only used when they are defined by crystal symmetry. An approximate (isotropic) treatment of cell esds is used for estimating esds involving l.s. planes.

A slightly large prolate is evident in thermal ellipsoid of $\mathrm{C} 10$ which results from what is presumed to be a partial population of I due to a trace impurity in this position. Refinement with a partial population of I in this position $(<4 \%)$ did not significantly improve the overall refinement and was not included as a part of the final refinement. Hydrogen bonding between F1 and N2 is also evident and summarized in Table 16. 
Table 12. Atomic coordinates ( $\left.\mathrm{x} 10^{4}\right)$ and equivalent isotropic displacement parameters $\left(\AA^{2} \times 10^{3}\right)$ for 4. $U(\mathrm{eq})$ is defined as one third of the trace of the orthogonalized $U^{i j}$ tensor.

\begin{tabular}{lllrl}
\hline & \multicolumn{1}{c}{$\mathrm{x}$} & \multicolumn{1}{c}{$\mathrm{y}$} & $\mathrm{z}$ & $\mathrm{U}(\mathrm{eq})$ \\
\hline $\mathrm{Pt}$ & & & & \\
$\mathrm{N}(1)$ & $5709(1)$ & 2500 & $1981(1)$ & $13(1)$ \\
$\mathrm{N}(2)$ & $4656(3)$ & $3563(2)$ & $1902(4)$ & $16(1)$ \\
$\mathrm{C}(10)$ & $5257(3)$ & 2500 & $4040(6)$ & $15(1)$ \\
$\mathrm{I}$ & $6825(3)$ & $3496(3)$ & $2203(5)$ & $22(1)$ \\
$\mathrm{C}(1)$ & $6194(1)$ & 2500 & $-549(1)$ & $25(1)$ \\
$\mathrm{C}(2)$ & $4353(3)$ & $4023(3)$ & $845(5)$ & $20(1)$ \\
$\mathrm{C}(3)$ & $3795(3)$ & $4813(3)$ & $971(6)$ & $23(1)$ \\
$\mathrm{C}(4)$ & $3552(3)$ & $5122(3)$ & $2242(6)$ & $22(1)$ \\
$\mathrm{C}(5)$ & $3848(3)$ & $4620(3)$ & $3387(5)$ & $18(1)$ \\
$\mathrm{C}(6)$ & $3627(4)$ & $4888(3)$ & $4729(6)$ & $24(1)$ \\
$\mathrm{C}(7)$ & $3938(4)$ & $4382(3)$ & $5796(6)$ & $26(1)$ \\
$\mathrm{C}(8)$ & $4493(4)$ & $3597(3)$ & $5585(5)$ & $22(1)$ \\
$\mathrm{C}(9)$ & $4712(3)$ & $3324(2)$ & $4298(4)$ & $15(1)$ \\
$\mathrm{B}$ & $4400(3)$ & $3833(3)$ & $3182(4)$ & $14(1)$ \\
$\mathrm{F}(1)$ & $7704(6)$ & 2500 & $6080(8)$ & $23(1)$ \\
$\mathrm{F}(2)$ & $6693(4)$ & 2500 & $6059(6)$ & $45(2)$ \\
$\mathrm{F}(3)$ & $8046(4)$ & 2500 & $4737(5)$ & $30(1)$ \\
& $8027(3)$ & $3270(3)$ & $6714(5)$ & $43(1)$ \\
\hline
\end{tabular}


Table 13. Bond lengths $[\AA]$ and angles $\left[{ }^{\circ}\right]$ for 4.

\begin{tabular}{|c|c|c|c|}
\hline Pt-N(2) & $2.135(5)$ & $\mathrm{C}(1)-\mathrm{N}(1)-\mathrm{C}(9)$ & $119.9(4)$ \\
\hline Pt-N(1)\#1 & $2.151(4)$ & $\mathrm{C}(1)-\mathrm{N}(1)-\mathrm{Pt}$ & $128.8(3)$ \\
\hline Pt-N(1) & $2.151(4)$ & $\mathrm{C}(9)-\mathrm{N}(1)-\mathrm{Pt}$ & $110.8(3)$ \\
\hline $\mathrm{Pt}-\mathrm{C}(10) \# 1$ & $2.151(4)$ & $\mathrm{C}(8) \# 1-\mathrm{N}(2)-\mathrm{C}(8)$ & $114.0(4)$ \\
\hline Pt-C(10) & $2.151(4)$ & $\mathrm{C}(8) \# 1-\mathrm{N}(2)-\mathrm{Pt}$ & $108.6(3)$ \\
\hline Pt-I & $2.5978(5)$ & $\mathrm{C}(8)-\mathrm{N}(2)-\mathrm{Pt}$ & $108.6(3)$ \\
\hline $\mathrm{N}(1)-\mathrm{C}(1)$ & $1.320(6)$ & $\mathrm{C}(8) \# 1-\mathrm{N}(2)-\mathrm{F}(1)$ & $103.8(3)$ \\
\hline $\mathrm{N}(1)-\mathrm{C}(9)$ & $1.378(6)$ & $\mathrm{C}(8)-\mathrm{N}(2)-\mathrm{F}(1)$ & $103.8(3)$ \\
\hline $\mathrm{N}(2)-\mathrm{C}(8) \# 1$ & $1.462(5)$ & $\mathrm{Pt}-\mathrm{N}(2)-\mathrm{F}(1)$ & $118.2(2)$ \\
\hline $\mathrm{N}(2)-\mathrm{C}(8)$ & $1.462(5)$ & $\mathrm{C}(8) \# 1-\mathrm{N}(2)-\mathrm{H}(11)$ & 108.5 \\
\hline$N(2)-F(1)$ & $2.821(7)$ & $\mathrm{C}(8)-\mathrm{N}(2)-\mathrm{H}(11)$ & 108.5 \\
\hline $\mathrm{N}(2)-\mathrm{H}(11)$ & 0.9300 & $\mathrm{Pt}-\mathrm{N}(2)-\mathrm{H}(11)$ & 108.5 \\
\hline $\mathrm{C}(10)-\mathrm{H}(10 \mathrm{~A})$ & 0.9800 & $\mathrm{~F}(1)-\mathrm{N}(2)-\mathrm{H}(11)$ & 9.7 \\
\hline $\mathrm{C}(10)-\mathrm{H}(10 \mathrm{~B})$ & 0.9800 & $\mathrm{Pt}-\mathrm{C}(10)-\mathrm{H}(10 \mathrm{~A})$ & 109.5 \\
\hline $\mathrm{C}(10)-\mathrm{H}(10 \mathrm{C})$ & 0.9800 & $\mathrm{Pt}-\mathrm{C}(10)-\mathrm{H}(10 \mathrm{~B})$ & 109.5 \\
\hline $\mathrm{C}(1)-\mathrm{C}(2)$ & $1.412(7)$ & Pt-C(10)-H(10C) & 109.5 \\
\hline $\mathrm{C}(1)-\mathrm{H}(1)$ & 0.9500 & $\mathrm{~N}(1)-\mathrm{C}(1)-\mathrm{C}(2)$ & $122.4(5)$ \\
\hline $\mathrm{C}(2)-\mathrm{C}(3)$ & $1.383(8)$ & $\mathrm{N}(1)-\mathrm{C}(1)-\mathrm{H}(1)$ & 118.8 \\
\hline $\mathrm{C}(2)-\mathrm{H}(2)$ & 0.9500 & $\mathrm{C}(2)-\mathrm{C}(1)-\mathrm{H}(1)$ & 118.8 \\
\hline$C(3)-C(4)$ & $1.420(7)$ & $\mathrm{C}(3)-\mathrm{C}(2)-\mathrm{C}(1)$ & $119.3(5)$ \\
\hline $\mathrm{C}(3)-\mathrm{H}(3)$ & 0.9500 & $\mathrm{C}(3)-\mathrm{C}(2)-\mathrm{H}(2)$ & 120.3 \\
\hline $\mathrm{C}(4)-\mathrm{C}(9)$ & $1.414(6)$ & $\mathrm{C}(1)-\mathrm{C}(2)-\mathrm{H}(2)$ & 120.3 \\
\hline$C(4)-C(5)$ & $1.423(7)$ & $\mathrm{C}(2)-\mathrm{C}(3)-\mathrm{C}(4)$ & $118.9(4)$ \\
\hline$C(5)-C(6)$ & $1.368(8)$ & $\mathrm{C}(2)-\mathrm{C}(3)-\mathrm{H}(3)$ & 120.5 \\
\hline $\mathrm{C}(5)-\mathrm{H}(5)$ & 0.9500 & $\mathrm{C}(4)-\mathrm{C}(3)-\mathrm{H}(3)$ & 120.5 \\
\hline $\mathrm{C}(6)-\mathrm{C}(7)$ & $1.414(7)$ & $\mathrm{C}(9)-\mathrm{C}(4)-\mathrm{C}(3)$ & $118.5(4)$ \\
\hline $\mathrm{C}(6)-\mathrm{H}(6)$ & 0.9500 & $C(9)-C(4)-C(5)$ & $118.9(4)$ \\
\hline$C(7)-C(8)$ & $1.373(6)$ & $C(3)-C(4)-C(5)$ & $122.6(4)$ \\
\hline $\mathrm{C}(7)-\mathrm{H}(7)$ & 0.9500 & $C(6)-C(5)-C(4)$ & $120.1(4)$ \\
\hline $\mathrm{C}(8)-\mathrm{C}(9)$ & $1.409(6)$ & $\mathrm{C}(6)-\mathrm{C}(5)-\mathrm{H}(5)$ & 119.9 \\
\hline $\mathrm{B}-\mathrm{F}(3)$ & $1.381(6)$ & $\mathrm{C}(4)-\mathrm{C}(5)-\mathrm{H}(5)$ & 119.9 \\
\hline $\mathrm{B}-\mathrm{F}(3) \# 1$ & $1.381(6)$ & $C(5)-C(6)-C(7)$ & $120.7(5)$ \\
\hline $\mathrm{B}-\mathrm{F}(1)$ & $1.398(10)$ & $\mathrm{C}(5)-\mathrm{C}(6)-\mathrm{H}(6)$ & 119.6 \\
\hline \multirow[t]{2}{*}{$\mathrm{B}-\mathrm{F}(2)$} & $1.414(9)$ & $\mathrm{C}(7)-\mathrm{C}(6)-\mathrm{H}(6)$ & 119.6 \\
\hline & & $\mathrm{C}(8)-\mathrm{C}(7)-\mathrm{C}(6)$ & $120.1(5)$ \\
\hline N(2)-Pt-N(1)\#1 & $80.62(13)$ & $\mathrm{C}(8)-\mathrm{C}(7)-\mathrm{H}(7)$ & 119.9 \\
\hline $\mathrm{N}(2)-\mathrm{Pt}-\mathrm{N}(1)$ & $80.62(13)$ & $\mathrm{C}(6)-\mathrm{C}(7)-\mathrm{H}(7)$ & 119.9 \\
\hline N(1)\#1-Pt-N(1) & $94.67(19)$ & $\mathrm{C}(7)-\mathrm{C}(8)-\mathrm{C}(9)$ & $120.2(4)$ \\
\hline N(2)-Pt-C(10)\#1 & $96.46(17)$ & $\mathrm{C}(7)-\mathrm{C}(8)-\mathrm{N}(2)$ & $121.7(4)$ \\
\hline $\mathrm{N}(1) \# 1-\mathrm{Pt}-\mathrm{C}(10) \# 1$ & $89.03(14)$ & $\mathrm{C}(9)-\mathrm{C}(8)-\mathrm{N}(2)$ & 118.1(4) \\
\hline N(1)-Pt-C(10)\#1 & $174.84(15)$ & $\mathrm{N}(1)-\mathrm{C}(9)-\mathrm{C}(8)$ & $119.2(3)$ \\
\hline N(2)-Pt-C(10) & $96.46(17)$ & $\mathrm{N}(1)-\mathrm{C}(9)-\mathrm{C}(4)$ & $120.9(4)$ \\
\hline N(1)\#1-Pt-C(10) & $174.84(15)$ & $\mathrm{C}(8)-\mathrm{C}(9)-\mathrm{C}(4)$ & $119.9(4)$ \\
\hline $\mathrm{N}(1)-\mathrm{Pt}-\mathrm{C}(10)$ & $89.03(14)$ & $\mathrm{F}(3)-\mathrm{B}-\mathrm{F}(3) \# 1$ & 112.1(7) \\
\hline $\mathrm{C}(10) \# 1-\mathrm{Pt}-\mathrm{C}(10)$ & $87.1(2)$ & $\mathrm{F}(3)-\mathrm{B}-\mathrm{F}(1)$ & $109.3(4)$ \\
\hline $\mathrm{N}(2)-\mathrm{Pt}-\mathrm{I}$ & 177.94(13) & $\mathrm{F}(3) \# 1-\mathrm{B}-\mathrm{F}(1)$ & 109.3(4) \\
\hline N(1)\#1-Pt-I & $98.00(10)$ & $\mathrm{F}(3)-\mathrm{B}-\mathrm{F}(2)$ & $108.7(4)$ \\
\hline N(1)-Pt-I & $98.01(10)$ & $\mathrm{F}(3) \# 1-\mathrm{B}-\mathrm{F}(2)$ & $108.7(4)$ \\
\hline C(10)\#1-Pt-I & $85.02(15)$ & $F(1)-B-F(2)$ & $108.7(6)$ \\
\hline C(10)-Pt-I & $85.02(15)$ & B-F(1)-N(2) & $135.6(5)$ \\
\hline
\end{tabular}

Symmetry transformations used to generate equivalent atoms:

$\# 1 \mathrm{x},-\mathrm{y}+1 / 2, \mathrm{z}$ 
Table 14. Anisotropic displacement parameters $\left(\AA^{2} \times 10^{3}\right)$ for 4 . The anisotropic displacement factor exponent takes the form: $-2 \pi^{2}\left[\mathrm{~h}^{2} \mathrm{a}^{* 2} \mathrm{U}^{11}+\ldots+2 \mathrm{~h} \mathrm{k} \mathrm{a}^{*} \mathrm{~b}^{*} \mathrm{U}^{12}\right]$

\begin{tabular}{lcccccc}
\hline & $\mathrm{U}^{11}$ & $\mathrm{U}^{22}$ & $\mathrm{U}^{33}$ & $\mathrm{U}^{23}$ & $\mathrm{U}^{13}$ & $\mathrm{U}^{12}$ \\
\hline $\mathrm{Pt}$ & $15(1)$ & $10(1)$ & $15(1)$ & 0 & $3(1)$ & 0 \\
$\mathrm{~N}(1)$ & $18(2)$ & $12(1)$ & $17(2)$ & $1(1)$ & $-1(1)$ & $0(1)$ \\
$\mathrm{N}(2)$ & $8(2)$ & $17(2)$ & $20(2)$ & 0 & $0(1)$ & 0 \\
$\mathrm{C}(10)$ & $24(2)$ & $9(1)$ & $34(2)$ & $-13(1)$ & $-22(2)$ & $9(1)$ \\
$\mathrm{I}$ & $38(1)$ & $17(1)$ & $21(1)$ & 0 & $11(1)$ & 0 \\
$\mathrm{C}(1)$ & $17(2)$ & $19(2)$ & $24(2)$ & $4(1)$ & $-4(1)$ & $-1(1)$ \\
$\mathrm{C}(2)$ & $20(2)$ & $18(2)$ & $31(2)$ & $6(2)$ & $-4(2)$ & $3(1)$ \\
$\mathrm{C}(3)$ & $17(2)$ & $13(1)$ & $36(3)$ & $2(2)$ & $1(2)$ & $1(1)$ \\
$\mathrm{C}(4)$ & $14(2)$ & $12(1)$ & $27(2)$ & $-2(1)$ & $3(1)$ & $0(1)$ \\
$\mathrm{C}(5)$ & $21(2)$ & $16(2)$ & $34(3)$ & $-6(2)$ & $11(2)$ & $2(1)$ \\
$\mathrm{C}(6)$ & $29(2)$ & $21(2)$ & $27(3)$ & $-8(2)$ & $10(2)$ & $-2(2)$ \\
$\mathrm{C}(7)$ & $27(2)$ & $16(2)$ & $22(2)$ & $-3(1)$ & $5(2)$ & $-2(1)$ \\
$\mathrm{C}(8)$ & $15(2)$ & $11(1)$ & $19(2)$ & $-2(1)$ & $1(1)$ & $-1(1)$ \\
$\mathrm{C}(9)$ & $11(1)$ & $13(1)$ & $20(2)$ & $-1(1)$ & $2(1)$ & $-1(1)$ \\
$\mathrm{B}$ & $25(3)$ & $31(3)$ & $11(3)$ & 0 & $-2(2)$ & 0 \\
$\mathrm{~F}(1)$ & $24(2)$ & $89(5)$ & $21(2)$ & 0 & $-5(2)$ & 0 \\
$\mathrm{~F}(2)$ & $31(2)$ & $40(3)$ & $20(2)$ & 0 & $5(2)$ & 0 \\
$\mathrm{~F}(3)$ & $45(2)$ & $39(2)$ & $46(2)$ & $-22(2)$ & $8(2)$ & $-9(2)$ \\
& & & & & & \\
\hline
\end{tabular}

Table 15. Hydrogen coordinates ( $\left.\times 10^{4}\right)$ and isotropic displacement parameters $\left(\AA^{2} \times 10^{3}\right)$ for 4 .

\begin{tabular}{lrrrr}
\hline & $\mathrm{x}$ & $\mathrm{y}$ & $\mathrm{z}$ & $\mathrm{U}(\mathrm{eq})$ \\
\hline $\mathrm{H}(11)$ & 5804 & 2500 & 4584 & 18 \\
$\mathrm{H}(10 \mathrm{~A})$ & 6550 & 4095 & 2061 & 33 \\
$\mathrm{H}(10 \mathrm{~B})$ & 7334 & 3387 & 1536 & 33 \\
$\mathrm{H}(10 \mathrm{C})$ & 7099 & 3459 & 3112 & 33 \\
$\mathrm{H}(1)$ & 4516 & 3815 & -31 & 24 \\
$\mathrm{H}(2)$ & 3588 & 5129 & 190 & 28 \\
$\mathrm{H}(3)$ & 3194 & 5662 & 2347 & 26 \\
$\mathrm{H}(5)$ & 3263 & 5419 & 4884 & 29 \\
$\mathrm{H}(6)$ & 3780 & 4561 & 6688 & 31 \\
$\mathrm{H}(7)$ & 4714 & 3258 & 6335 & 26 \\
\hline
\end{tabular}

Table 16. Hydrogen bonds for $4\left[\AA\right.$ and $\left.{ }^{\circ}\right]$.

\begin{tabular}{lcccc}
\hline $\mathrm{D}-\mathrm{H} \ldots \mathrm{A}$ & $\mathrm{d}(\mathrm{D}-\mathrm{H})$ & $\mathrm{d}(\mathrm{H} \ldots \mathrm{A})$ & $\mathrm{d}(\mathrm{D} \ldots \mathrm{A})$ & $<(\mathrm{DHA})$ \\
\hline $\mathrm{N}(2)-\mathrm{H}(11) \ldots \mathrm{F}(1)$ & 0.93 & 1.91 & $2.821(7)$ & 165.6
\end{tabular}

Symmetry transformations used to generate equivalent atoms:

$\# 1 \mathrm{x},-\mathrm{y}+1 / 2, \mathrm{z}$ 